\title{
The Propagation Depth of Local Consistency
}

\author{
Christoph Berkholz \\ RWTH Aachen University, Aachen, Germany
}

\begin{abstract}
We establish optimal bounds on the number of nested propagation steps in $k$-consistency tests. It is known that local consistency algorithms such as arc-, path- and $k$-consistency are not efficiently parallelizable. Their inherent sequential nature is caused by long chains of nested propagation steps, which cannot be executed in parallel. This motivates the question "What is the minimum number of nested propagation steps that have to be performed by $k$-consistency algorithms on (binary) constraint networks with $n$ variables and domain size $d$ ?"

It was known before that 2-consistency requires $\Omega(n d)$ and 3-consistency requires $\Omega\left(n^{2}\right)$ sequential propagation steps. We answer the question exhaustively for every $k \geq 2$ : there are binary constraint networks where any $k$-consistency procedure has to perform $\Omega\left(n^{k-1} d^{k-1}\right)$ nested propagation steps before local inconsistencies were detected. This bound is tight, because the overall number of propagation steps performed by $k$-consistency is at most $n^{k-1} d^{k-1}$.
\end{abstract}

\section{Introduction}

A constraint network $(X, D, C)$ consists of a set $X$ of $n$ variables over a domain $D$ of size $d$ and a set of constraints $C$ that restrict possible assignments of the variables. The constraint satisfaction problem (CSP) is to find an assignment of the variables with values from $D$ such that all constraints are satisfied. The constraint satisfaction problem can be solved in exponential time by exhaustive search over all possible assignments. Constraint propagation is a technique to speed up the exhaustive search by restricting the search space in advance. This is done by iteratively propagating new constraints that follow from previous ones. Most notably, in local consistency algorithms the overall goal is to propagate new constraints to achieve some kind of consistency on small parts of the constraint network. Additionally, if local inconsistencies were detected, it follows that the constraint network is also globally inconsistent and hence unsatisfiable.

The $k$-consistency test $[\underline{8}$ is a well-known local consistency technique, which enforces that every satisfying $(k-1)$-partial assignment can be extended to a satisfying $k$-partial assignment. At the beginning, all partial assignments that violate a constraint were marked as inconsistent. Then the following inference rule is applied iteratively:

If $h$ is a consistent $\ell$-partial assignment $(\ell<k)$ for which there exists a variable $x \in X$ such that $h \cup\{x \mapsto a\}$ is inconsistent for all $a \in D$, then mark $h$ and all its extensions as inconsistent. 
After at most $n^{k-1} d^{k-1}$ propagation steps this procedure stops. If the empty assignment becomes inconsistent, we say that (strong) $k$-consistency cannot be established. In this case we know that the constraint network is globally inconsistent. Otherwise, if $k$-consistency can be established, we can use the propagated constraints to restrict the search space for a subsequent exhaustive search. There are several different $k$-consistency algorithms in the literature, especially for $k=2$ (arc consistency) and $k=3$ (path consistency), which all follow this propagation scheme. The main difference between these algorithms are the underlying data structure and the order in which they apply the propagation rule. It seems plausible to apply the propagation rule in parallel in order to detect local inconsistencies in different parts of the constraint network at the same time. Indeed, this intuition has been used to design parallel arc and path consistency algorithms [15]16. On the other hand, the $k$-consistency test is known to be PTIME-complete 1011 and hence not efficiently parallelizable (unless $\mathrm{NC}=\mathrm{PTIME}$ ). The main bottleneck for parallel approaches are the sequential dependencies in the propagation rule: some assignments will be marked as inconsistent after some other assignments became inconsistent.

For 2-consistency the occurrence of long chains of sequential dependencies has been observed very early [6] and was recently studied in depth in [4. There are simple constraint networks for which 2-consistency requires $\Omega(n d)$ nested propagation steps. Ladkin and Maddux 14] used algebraic techniques to show that 3-consistency requires $\Omega\left(n^{2}\right)$ nested propagation steps on binary constraint networks with constant domain. We extend these previous results and obtain a complete picture of the propagation depth of $k$-consistency. Our main result (Theorem 11) states that for every constant $k \geq 2$ and given integers $n$, $d$ there is a constraint network with $n$ variables and domain size $d$ such that every $k$-consistency algorithm has to perform $\Omega\left(n^{k-1} d^{k-1}\right)$ nested propagation steps. This lower bound is optimal as it is matched by the trivial upper bound $n^{k-1} d^{k-1}$ on the overall number of propagation steps. It follows that every parallel propagation algorithm for $k$-consistency has a worst case time complexity of $\Omega\left(n^{k-1} d^{k-1}\right)$. Since the best-known running time of a sequential algorithm for $k$-consistency is $O\left(n^{k} d^{k}\right)[5]$ it follows that no significant improvement over the sequential algorithm is possible.

\section{Preliminaries}

As first pointed out by Feder and Vardi [7] the CSP is equivalent to the structure homomorphism problem where two finite relational structures $A$ and $B$ are given as input. The universe $V(\mathrm{~A})$ of structure $\mathrm{A}$ corresponds to the set of variables $X$ and the universe $V(\mathrm{~B})$ of structure $\mathrm{B}$ corresponds to the domain $D$. The constraints are encoded into relations such that every homomorphism from $A$ to $\mathrm{B}$ corresponds to a solution of the CSP. For the rest of this paper we mainly stick to this definition as it is more convenient to us. In fact, our main result benefits to a large extend from the fruitful connection between these two viewpoints. 
In the introduction we have presented $k$-consistency as a propagation procedure on constraint networks. Below we restate the definition in terms of a formal inference system (which is inspired by the proof system in [1] and is a generalization of (4)). This view allows us to gain insight into the structure of the propagation process and to formally state our main theorem afterwards. At the end of this section we provide a third characterization of $k$-consistency in terms of the existential pebble game, which is the tool of our choice in the proof of the main theorem.

\subsection{CSP-refutations}

Given two $\sigma$-structures $\mathrm{A}$ and $\mathrm{B}$, every line of our derivation system is a partial mapping from $V(\mathrm{~A})$ to $V(\mathrm{~B})$. The axioms are all partial mappings $p: V(\mathrm{~A}) \rightarrow$ $V(\mathrm{~B})$ that are not partial homomorphisms. We have the following derivation rule to derive a new inconsistent assignment $p$. For all partial mappings $p_{i}^{\prime} \subseteq p$, $x \in V(\mathrm{~A})$ and $V(\mathrm{~B})=\left\{a_{1}, \ldots, a_{n}\right\}:$

$$
\frac{p_{1}^{\prime} \cup\left\{x \mapsto a_{1}\right\} \quad \cdots \quad p_{n}^{\prime} \cup\left\{x \mapsto a_{n}\right\}}{p}
$$

A CSP-derivation of $p$ is a sequence $\left(p_{1}, \ldots, p_{\ell}=p\right)$ such that every $p_{i}$ is either an axiom or derived from lines $p_{j}, j<i$, via the derivation rule (1). A CSPrefutation is a CSP-derivation of $\emptyset$. Every derivation of $p$ can naturally be seen as a directed acyclic graph (dag) where the nodes are labeled with lines from the derivation, one node of in-degree 0 is labeled with $p$ and all nodes of out-degree 0 are labeled with axioms. If $p_{i}$ is derived from $p_{j_{1}}, \ldots, p_{j_{n}}$ using (1), then there is an arc from $p_{i}$ to each $p_{j_{1}}, \ldots, p_{j_{n}}$.

Given a CSP-derivation $P$, we let $\operatorname{Prop}(P)$ be the set of propagated mappings $p \in P$, i. e. all lines in the derivation that are not axioms. We define the width of a derivation $P$ to be $\operatorname{width}(P)=\left.\max _{p \in \operatorname{Prop}(P)}|p|\right|^{1}$ Furthermore, $\operatorname{depth}(P)$ denotes the depth of $P$ which is the number of edges on the longest path in the dag associated with $P$. This measure characterizes the maximum number of nested propagation steps in $P$. Since CSP-derivations model the propagation process mentioned in the introduction, there is a CSP-refutation of width $k-1$ if and only if $k$-consistency cannot be established.

Furthermore, every propagation algorithm produces some CSP-derivation $P$. The total number of propagation steps performed by this algorithm is $|\operatorname{Prop}(P)|$ and the maximum number of nested propagation steps is $\operatorname{depth}(P)$. Let $\mathrm{A}$ and $\mathrm{B}$ be two relational structures such that $k$-consistency cannot be established. We define the propagation depth $\operatorname{depth}^{k}(\mathrm{~A}, \mathrm{~B}):=\min _{P} \operatorname{depth}(P)$ where the minimum is taken over all CSP-refutations $P$ of width at most $k-1$. Hence, the $\operatorname{depth}^{k}(\mathrm{~A}, \mathrm{~B}) \leq|V(\mathrm{~A})|^{k-1}|V(\mathrm{~B})|^{k-1}$ is the number of sequential propagation steps that have to be performed by any sequential or parallel propagation algorithm for $k$-consistency.

\footnotetext{
${ }^{1}$ Note that this implies $|p| \leq \operatorname{width}(P)+1$ for all axioms $p$ used in the derivation $P$. However, the size of the axioms can always be bounded by the maximum arity of the relations in $A$ and $B$.
} 


\subsection{Results and Related Work}

Our main theorem is a tight lower bound on the propagation depth.

Theorem 1. For every integer $k \geq 2$ there exists a constant $\varepsilon>0$ and two positive integers $n_{0}, m_{0}$ such that for every $n \geq n_{0}$ and $m \geq m_{0}$ there exist two binary structures $\mathrm{A}_{n}$ and $\mathrm{B}_{m}$ with $\left|V\left(\mathrm{~A}_{n}\right)\right|=n$ and $\left|V\left(\mathrm{~B}_{m}\right)\right|=m$ such that $\operatorname{depth}^{k}\left(\mathrm{~A}_{n}, \mathrm{~B}_{m}\right) \geq \varepsilon n^{k-1} m^{k-1}$.

We are aware of two particular cases that have been discovered earlier. First, for the case $k=2$ (arc consistency) the theorem can be shown by rather simple examples that occurred very early in the AI-community. The structure of this exceptional case is discussed in deep in a joint work of Oleg Verbitsky and the author of this paper [4. Second, for $k=3$ Ladkin and Maddux [14] showed that there is a fixed finite binary structure $B$ and an infinite sequence of binary structures $\mathrm{A}_{i}$ such that $\operatorname{depth}^{3}\left(\mathrm{~A}_{i}, \mathrm{~B}\right)=\Omega\left(\left|V\left(\mathrm{~A}_{i}\right)\right|^{2}\right)$. They used this result to argue that every parallel propagation algorithm for path consistency needs at least a quadratic number of steps. This is tight only for fixed structures B, Theorem 1 extends their result to the case when B is also given as input.

Other related results investigate the decision complexity of the $k$-consistency test. To address this more general question one analyzes the computational complexity of the following decision problem.

\section{$k$-Cons}

Input: Two binary relational structures $\mathrm{A}$ and $\mathrm{B}$.

Question: Can $k$-consistency be established for A and B?

Kasif [10] showed that 2-Cons is complete for PTIME under LOGSPACE reductions. Kolaitis and Panttaja [1] extended this result to every fixed $k \geq 2$. Moreover, they established that the problem is complete for EXPTIME if $k$ is part of the input. In 3 the author showed that $k$-Cons cannot be decided in $O\left(n^{\frac{k-3}{12}}\right)$ on deterministic multi-tape Turing machines, where $n$ is the overall input size. Hence, any algorithm solving $k$-Cons (regardless of whether it performs constraint propagation or not) cannot be much faster than the standard propagation approach. It also follows from this result that, parameterized by the number of pebbles $k, k$-Cons is is complete for the parameterized complexity class XP. It is also worth noting that Gaspers and Szeider [9] investigated the parameterized complexity of other parameterized problems related to $k$-consistency.

\subsection{The Existential Pebble Game}

In this paragraph we introduce a third view on the $k$-consistency heuristic in terms of a combinatorial pebble game. The existential k-pebble game [12] is played by two players Spoiler and Duplicator on two relational structures A and B. There are $k$ pairs of pebbles $\left(p_{1}, q_{1}\right), \ldots,\left(p_{k}, q_{k}\right)$ and during the game Spoiler moves the pebbles $p_{1}, \ldots, p_{k}$ to elements of $V(\mathrm{~A})$ and Duplicator moves 
the pebbles $q_{1}, \ldots, q_{k}$ to elements of $V(\mathrm{~B})$. At the beginning of the game, Spoiler places pebbles $p_{1}, \ldots, p_{k}$ on elements of $V(\mathrm{~A})$ and Duplicator answers by putting pebbles $q_{1}, \ldots, q_{k}$ on elements of $V(\mathrm{~B})$. In each further round Spoiler picks up a pebble pair $\left(p_{i}, q_{i}\right)$ and places $p_{i}$ on some element in $V(\mathrm{~A})$. Duplicator answers by moving the corresponding pebble $q_{i}$ to one element in $V(\mathrm{~B})$. Spoiler wins the game if he can reach a position where the mapping defined by $p_{i} \mapsto q_{i}$ is not a partial homomorphism from A to B.

The connection between the existential $k$-pebble game and the $k$-consistency heuristic was made by Kolaitis and Vardi [13. They showed that one can establish $k$-consistency by computing a winning strategy for Duplicator. Going a different way, the next lemma states that there is also a tight correspondence between Spoiler's strategy and CSP-refutations. The proof is a straightforward induction over the depth and given in Appendix A.1.

Lemma 2. Let $\mathrm{A}$ and $\mathrm{B}$ be two relational structures. There is a CSP-refutation for $\mathrm{A}$ and $\mathrm{B}$ of width $k-1$ and depth $d$ if and only if Spoiler has a strategy to win the existential $k$-pebble game on $\mathrm{A}$ and $\mathrm{B}$ within $d$ rounds.

Using this lemma it suffices to prove lower bounds on the number of rounds in the existential pebble game in order to prove Theorem 11. To argue about strategies in the existential pebble game we use the framework developed in [3]. We start with a formal definition of strategies for Duplicator.

Definition 3. A critical strategy for Duplicator in the existential k-pebble game on structures $\mathrm{A}$ and $\mathrm{B}$ is a nonempty family $\mathcal{H}$ of partial homomorphisms from $\mathrm{A}$ to $\mathrm{B}$ together with a set $\operatorname{crit}(\mathcal{H}) \subseteq \mathcal{H}$ of critical positions satisfying the following properties:

1. All critical positions are $(k-1)$-partial homomorphisms.

2. If $h \in \mathcal{H}$ and $g \subset h$, then $g \in \mathcal{H}$.

3. For every $g \in \mathcal{H} \backslash \operatorname{crit}(\mathcal{H}),|g|<k$, and every $x \in V(\mathrm{~A})$ there is an $a \in V(\mathrm{~B})$ such that $g \cup\{x \mapsto a\} \in \mathcal{H}$.

If $\operatorname{crit}(\mathcal{H})=\emptyset$, then $\mathcal{H}$ is a winning strategy.

The set $\mathcal{H}$ is the set of good positions for Duplicator (therefore they are all partial homomorphisms). Non-emptiness and the closure property (2.) ensure that $\mathcal{H}$ contains the start position $\emptyset$. Furthermore, the closure property guarantees that the current position remains a good position for Duplicator when Spoiler picks up pebbles. The extension property (3.) ensures that, from every non-critical position, Duplicator has an appropriate answer if Spoiler puts a free pebble on $x$. It follows that if there are no critical positions, then Duplicator can always answer accordingly and thus wins the game. Otherwise, if Spoiler reaches a critical position, then Duplicator may not have an appropriate answer and the game reaches a critical state. In the next lemma we describe how to use critical strategies to prove lower bounds on the number of rounds.

Lemma 4. If $\mathcal{H}_{1}, \ldots, \mathcal{H}_{l}$ is a sequence of critical strategies on the same pair of structures and for all $i<l$ and all $p \in \operatorname{crit}\left(\mathcal{H}_{i}\right)$ it holds that $p \in \mathcal{H}_{j} \backslash \operatorname{crit}\left(\mathcal{H}_{j}\right)$ for some $j \leq i+1$, then Duplicator wins the l-round existential $k$-pebble game. 
Proof. Starting with $i=1$, Duplicator answers according to the extension property of $\mathcal{H}_{i}$, if the current position $p$ is non-critical in $\mathcal{H}_{i}$. Otherwise, $p$ is noncritical in $\mathcal{H}_{j}$ for some $j \leq i+1$ and Duplicator answers according to the extension property of $\mathcal{H}_{j}$. This allows Duplicator to survive for at least $l$ rounds.

The two structures A and B we construct are vertex colored graphs. They are built out of smaller graphs, called gadgets. Every gadget $Q$ consists of two graphs $Q_{S}$ and $Q_{D}$ for Spoiler's and Duplicator's side, respectively. Hence, $Q_{S}$ and $Q_{D}$ will be subgraphs of A and B in the end. The gadgets contain boundary vertices, which are the vertices shared with other gadgets. To combine two strategies on two connected gadgets we need to ensure that the strategies agree on the boundary of the gadgets. Formally, let a boundary function of a strategy $\mathcal{H}$ on a gadget $Q$ be a mapping $\beta$ from the boundary of $Q_{S}$ to the boundary of $Q_{D}$ such that $\beta(z)=h(z)$ for all $h \in \mathcal{H}$ and all $z$ in the domain of $\beta$ and $h$. We say that two strategies $\mathcal{G}$ and $\mathcal{H}$ on gadgets $Q$ and $Q^{\prime}$ are connectable, if their boundary functions agree on the common boundary vertices of $Q$ and $Q^{\prime}$. If $\mathcal{G}$ and $\mathcal{H}$ are two connectable critical strategies on gadgets $Q=\left(Q_{S}, Q_{D}\right)$ and $Q^{\prime}=\left(Q_{S}^{\prime}, Q_{D}^{\prime}\right)$ it is not hard to see that the composition

$$
\mathcal{G} \uplus \mathcal{H}=\{g \cup h \mid g \in \mathcal{G}, h \in \mathcal{H}\}
$$

is a critical strategy on $Q_{S} \cup Q_{S}^{\prime}$ and $Q_{D} \cup Q_{D}^{\prime}$ with $\operatorname{crit}(\mathcal{G} \uplus \mathcal{H})=\operatorname{crit}(\mathcal{G}) \cup \operatorname{crit}(\mathcal{H})$. Intuitively, playing according to the strategy $\mathcal{G} \uplus \mathcal{H}$ on $Q$ and $Q^{\prime}$ means that Duplicator uses strategy $\mathcal{G}$ on $Q$ and strategy $\mathcal{H}$ on $Q^{\prime}$.

\section{The Construction}

\subsection{Overview of the Construction}

In this section we prove Theorem 1 for $k \geq 3$. We let $\mathrm{k}:=k-1 \geq 2$ and construct two vertex colored graphs $\mathrm{A}_{n}$ and $\mathrm{B}_{m}$ with $O(n)$ and $O(m)$ vertices such that Spoiler needs $\Omega\left(n^{\mathrm{k}} m^{\mathrm{k}}\right)$ rounds to win the existential $(\mathrm{k}+1)$-pebble game. We color the vertices of both graphs such that the colors partition the vertex set into independent sets, i.e. every vertex gets one color and there is no edge between vertices of the same color. The basic building blocks in our construction are sets of vertices which allow to store $n^{\mathrm{k}} m^{\mathrm{k}}$ partial homomorphisms with $\mathrm{k}$ pebbles.

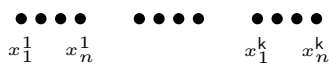

$\mathrm{A}_{n}$

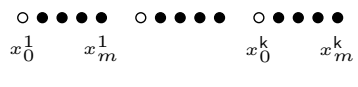

$\mathrm{B}_{m}$

Fig. 1. Basic vertex blocks. Two vertices $x_{j}^{i}$ and $x_{j^{\prime}}^{i^{\prime}}$ get the same color iff $i=i^{\prime}$.

We introduce vertices $x_{j}^{i}(i \in[\mathrm{k}], j \in[n])$ in $\mathrm{A}_{n}$ and vertices $x_{j}^{i}(i \in[\mathrm{k}]$, $j \in[m] \cup\{0\})$ in $\mathrm{B}_{m}$. For every $i \in[\mathrm{k}]$ the vertices $x_{j}^{i}$ form a block and are 
colored with the same color (say $P_{x^{i}}$ ), which is different from any other color in the entire construction. The vertices $x_{0}^{i}$ in structure $\mathrm{B}_{m}$ play a special role in our construction and are visualized by $\circ$ instead of $\bullet$ in the pictures. However, they are colored with the same color $P_{x^{i}}$ as the other vertices $x_{j}^{i}$. Because of the coloring, Duplicator has to answer with some $x_{j^{\prime}}^{i}$ whenever Spoiler pebbles a vertex $x_{j}^{i}$. Since there are $n m$ positions for one pebble pair on • vertices in one block, we get $n^{\mathrm{k}} m^{\mathrm{k}}$ positions if every block has exactly one pebble pair on - vertices. The $\circ$ vertices are used by Duplicator whenever Spoiler does not play the intended way. That is, if Spoiler pebbles a vertex in block $i$ that he is not supposed to pebble now, then Duplicator answers with $x_{0}^{i}$. The construction will have the property that this is always a good situation for Duplicator.

To describe pebble positions on such vertex blocks, we define mappings $\mathfrak{a}:[\mathrm{k}] \rightarrow[n]$ and $\mathfrak{b}:[\mathrm{k}] \rightarrow[m]$ and call the pebble position $\left\{\left(x_{\mathfrak{a}(i)}^{i}, x_{\mathfrak{b}(i)}^{i}\right) \mid i \in[\mathrm{k}]\right\}$ valid. If such valid position is on the board, then Duplicator answers with $x_{\mathfrak{b}(i)}^{i}$ if Spoiler pebbles $x_{\mathfrak{a}(i)}^{i}$ and with $x_{0}^{i}$ if Spoiler pebbles $x_{j}^{i}$ for some $j \neq \mathfrak{a}(i)$. We also need to name positions where Duplicator answers with $x_{0}^{i}$ for every vertex in block $i$ and let $T$ be the set of blocks where this happens. For $\mathfrak{a}:[\mathrm{k}] \rightarrow[n]$, $\mathfrak{b}:[\mathrm{k}] \rightarrow[m]$ and $T \subseteq[\mathrm{k}]$ we call $\mathfrak{q}=(\mathfrak{a}, \mathfrak{b}, T)$ a configuration. The configuration $\mathfrak{q}$ is valid if $T=\emptyset$ and invalid otherwise. For every configuration $\mathfrak{q}$ and a set of $x_{j}^{i}$ vertices as in Figure 1 we define the following homomorphism that describes Duplicator's behavior:

$$
h_{\mathfrak{q}}^{x}\left(x_{j}^{i}\right)=\left\{\begin{array}{l}
x_{\mathfrak{b}(i)}^{i}, \text { if } j=\mathfrak{a}(i) \text { and } i \notin T, \\
x_{0}^{i}, \text { otherwise. }
\end{array}\right.
$$

By $h_{\mathbf{0}}^{x}$ we denote the homomorphism $h_{\mathbf{0}}^{x}\left(x_{j}^{i}\right):=x_{0}^{i}$ for all $i \in[\mathrm{k}], j \in[n]$. We say that a position of (at most $\mathrm{k}+1$ ) pebble pairs on these vertices is invalid if it is a subset of $h_{\mathfrak{q}}^{x}$ for some invalid configuration $\mathfrak{q}$. For valid configurations $\mathfrak{q}=(\mathfrak{a}, \mathfrak{b}, \emptyset)$ we say "q on $x$ " to name the valid pebble position $\left\{\left(x_{\mathfrak{a}(i)}^{i}, x_{\mathfrak{b}(i)}^{i}\right) \mid i \in[\mathrm{k}]\right\}$. Note that valid pebble positions are not invalid ${ }^{2}$

In the entire construction there is one unique copy of the $x_{j}^{i}$-vertices, which are denoted by $x_{j}^{i}$. Our goal is to force Spoiler to pebble every valid position on $x$ before he wins the game. He is supposed to do so in a specific predefined order. To fix this order we define a bijection $\alpha$ between valid configurations $(\mathfrak{a}, \mathfrak{b}, \emptyset)$ and the numbers $0, \ldots, n^{\mathrm{k}} m^{\mathrm{k}}-1$ :

$$
\alpha(\mathfrak{q}):=m^{\mathrm{k}} \sum_{i=1}^{\mathrm{k}}(\mathfrak{a}(i)-1) n^{\mathrm{k}-i}+\sum_{i=1}^{\mathrm{k}}(\mathfrak{b}(i)-1) m^{\mathrm{k}-i} .
$$

Thus, $\alpha(\mathfrak{q})$ is the rank of the tuple $(\mathfrak{a}(1), \ldots, \mathfrak{a}(\mathrm{k}), \mathfrak{b}(1), \ldots, \mathfrak{b}(\mathbf{k}))$ in lexicographical order. If $\alpha(\mathfrak{q})<n^{\mathrm{k}} m^{\mathrm{k}}-1$, we define the successor $\mathfrak{q}^{+}=\left(\mathfrak{a}^{+}, \mathfrak{b}^{+}, \emptyset\right)$ to be the unique valid configuration satisfying $\alpha\left(\mathfrak{q}^{+}\right)=\alpha(\mathfrak{q})+1$. In the sequel we introduce gadgets to make sure that:

${ }^{2}$ There are pebble positions on the $x_{j}^{i}$ vertices that are neither valid nor invalid. However, such positions will not occur in our strategies. 
- Spoiler can reach the position $\alpha^{-1}(0)$ on $\times$ from $\emptyset$,

- Spoiler can reach $\alpha^{-1}(i+1)$ on $\mathrm{x}$ from $\alpha^{-1}(i)$ on $\mathrm{x}$ and

- Spoiler wins from $\alpha^{-1}\left(n^{\mathrm{k}} m^{\mathrm{k}}-1\right)$ on $\mathrm{x}$.

If we have these properties, we know that Spoiler has a winning strategy in the $(\mathrm{k}+1)$-pebble game. To show that Spoiler needs at least $n^{\mathrm{k}} m^{\mathrm{k}}$ rounds we argue that this is essentially the only way for Spoiler to win the game.

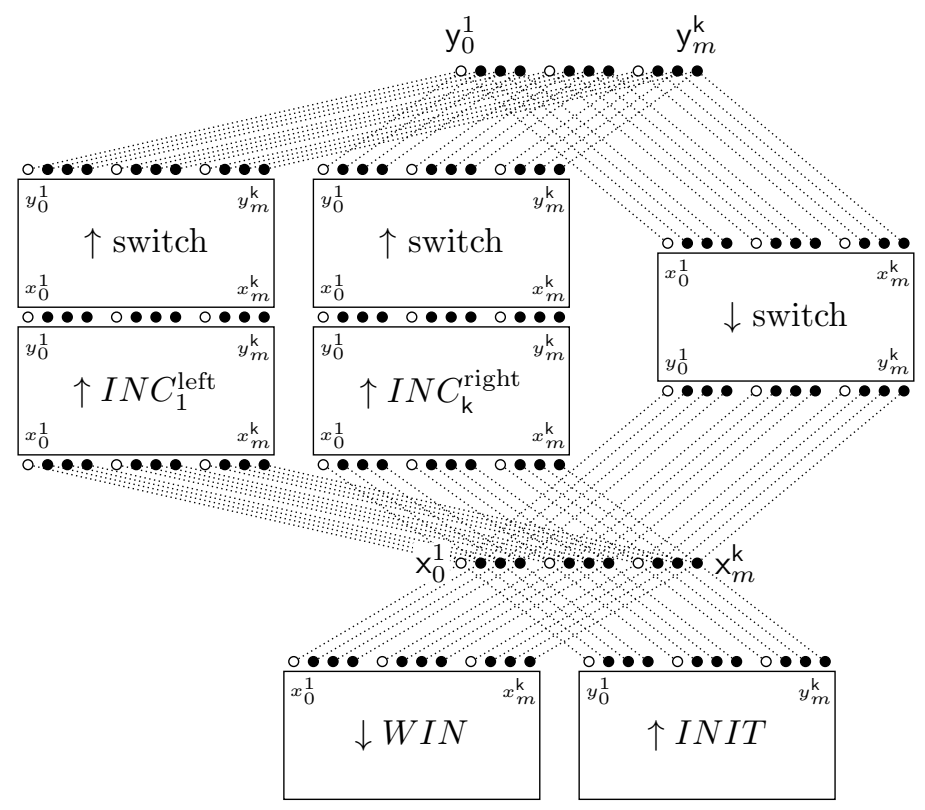

Fig. 2. The graph $\mathrm{B}_{m}$. The boundaries of the gadgets are connected as indicated by the dotted lines (which need to be contracted). The arrows point from the input to the output vertices of the gadgets.

We start with an overview of the gadgets and how they are glued together to form the structures $\mathrm{A}_{n}$ and $\mathrm{B}_{m}$. The boundary of our gadgets consists of input vertices and output vertices. For every gadget the set of input (output) vertices is a copy of the vertex set in Figure 1 and we write $x_{j}^{i}\left(y_{j}^{i}\right)$ to name them. This enables us to glue together the gadgets at their input and output vertices. The overall construction for the graph $\mathrm{B}_{m}$ is shown in Figure 2 The schema for $\mathrm{A}_{n}$ is similar, it contains Spoiler's side of the corresponding gadgets which are glued together the same way as in $\mathrm{B}_{m}$ (just replace $m$ by $n$ and drop the o vertices). There are four types of gadgets: the initialization gadget, the winning gadget, several increment gadgets and the switch.

The initialization gadget ensures that Spoiler can reach $\alpha^{-1}(0)$ on $\mathrm{x}$, i. e. the pebble position $\left\{\left(\mathrm{x}_{1}^{1}, \mathrm{x}_{1}^{1}\right), \ldots,\left(\mathrm{x}_{1}^{k}, \mathrm{x}_{1}^{k}\right)\right\}$. This gadget has only output boundary vertices and is used by Spoiler at the beginning of the game. There are increment 
gadgets $\mathrm{INC}_{i}^{\text {left }}$ and $\mathrm{INC}_{i}^{\text {right }}$ for all $i \in[\mathrm{k}]$. The input vertices of every increment gadget are identified with the $x$ vertices as depicted in Figure 2, The increment gadgets (all together) ensure that Spoiler can increment a configuration. More precisely, for every valid configuration $\mathfrak{q}$ with $\alpha(\mathfrak{q})<n^{\mathrm{k}} m^{\mathrm{k}}-1$, there is one increment gadget INC such that Spoiler can reach $\mathfrak{q}^{+}$on the output of INC from $\mathfrak{q}$ on the input. Every increment gadget is followed by a copy of the switch. The input of $2 k$ switches is identified with the output of the $2 k$ increment gadgets and the output of these switches is identified with a unique block of y-vertices and the input of one additional single switch (see Figure 2). The output of this switch is in turn identified with the unique block of $x$-vertices. The switches are used to perform the transition in the game from $\alpha^{-1}(i)$ on $x$ to $\alpha^{-1}(i+1)$ on $x$. Spoiler can pebble a valid position through one switch: from $\mathfrak{q}$ on the input of a switch Spoiler can reach $\mathfrak{q}$ on the output of that switch. Hence, Spoiler can simply pebble the incremented position $\alpha^{-1}(i+1)$ from the output of an increment gadget through two switches to the $\mathrm{x}$-block.

Finally, the winning gadget ensures that from $\alpha^{-1}\left(n^{\mathrm{k}} m^{\mathrm{k}}-1\right)$ on $\times$ Spoiler wins the game. The winning gadget has only input vertices, which are identified with the $\mathrm{x}$-vertices. From $\alpha^{-1}\left(n^{\mathrm{k}} m^{\mathrm{k}}-1\right)$ on the input, Spoiler can win the game by playing on this gadget. On the other hand, the gadget ensures that Spoiler can only win from $\alpha^{-1}\left(n^{\mathrm{k}} m^{\mathrm{k}}-1\right)$ on $\mathrm{x}$ and Duplicator does not lose from any other configuration on $\mathrm{x}$.

\subsection{The Gadgets}

We now describe the winning gadget and the increment gadgets in detail and provide strategies for Spoiler and Duplicator on them. Afterwards we briefly discuss the switch and the initialization gadget. In the next section we combine the partial strategies on the gadgets to prove Theorem 1.

The winning gadget is shown in Figure 3 . On Spoiler's side there is just one additional vertex $a$, which is connected to $x_{n}^{i}$ for all $i \in[\mathrm{k}]$. On Duplicator's side there are $\mathrm{k}$ additional vertices $a^{i}, i \in[\mathrm{k}]$. Every $a^{i}$ is connected to all input vertices except $x_{m}^{i}$. We use one new vertex color to color the vertex $a$ and all vertices $a_{i}$. From the position $\left\{\left(x_{n}^{1}, x_{m}^{1}\right), \ldots,\left(x_{n}^{k}, x_{m}^{k}\right)\right\}$ " $\alpha^{-1}\left(n^{\mathrm{k}} m^{\mathrm{k}}-1\right)$ on $x^{\text {" }}$ Spoiler wins the game by placing the $(\mathrm{k}+1)$ st pebble on $a$. Duplicator has to answer with some $a_{i}$ (because of the coloring). Since there is an edge between $x_{n}^{i}$ and $a$ in $\mathrm{WIN}_{S}$ but none between $x_{m}^{i}$ and $a_{i}$ in $\mathrm{WIN}_{D}$, Spoiler wins immediately. It is also not hard to see that for any other position where at least one pebble pair $\left(x_{n}^{j}, x_{m}^{j}\right)$ is missing Duplicator can survive by choosing $a_{j}$.

The increment gadgets enable Spoiler to reach the successor $\mathfrak{q}^{+}$from $\mathfrak{q}$. Recall that we identify every valid configuration $\mathfrak{q}=(\mathfrak{a}, \mathfrak{b}, \emptyset)$ with the tuple $(\mathfrak{a}(1), \ldots, \mathfrak{a}(\mathbf{k}), \mathfrak{b}(1), \ldots, \mathfrak{b}(\mathrm{k})) \in[n]^{k} \times[m]^{k}$ and define $\alpha(\mathfrak{q})$ to be the rank (from 0 to $n^{k} m^{k}-1$ ) of this tuple in lexicographical order. Let $\mathfrak{q}$ be a valid configuration with $\alpha(\mathfrak{q})<n^{\mathrm{k}} m^{\mathrm{k}}-1$ and successor $\mathfrak{q}^{+}=\left(\mathfrak{a}^{+}(1), \ldots, \mathfrak{a}^{+}(\mathrm{k}), \mathfrak{b}^{+}(1), \ldots, \mathfrak{b}^{+}(\mathbf{k})\right)$. We use two types of increment gadgets, left and right, depending on whether the left-hand side of the tuple changes after incrementation or not. There are $k$ 

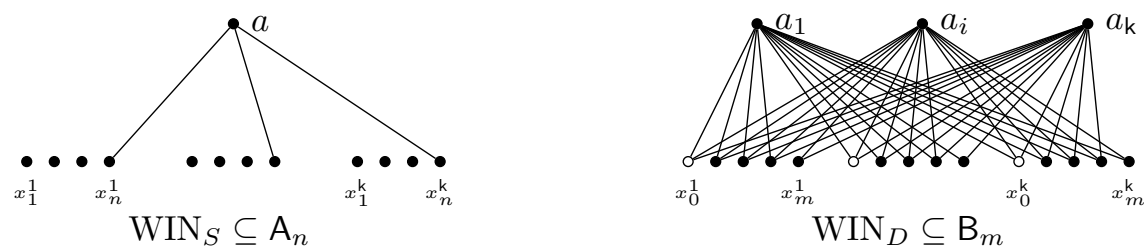

Fig. 3. The winning gadget.

increment gadgets of each type. Spoiler uses them depending on which position the last carryover occurs. If

$$
\begin{aligned}
& \mathfrak{q}=(\mathfrak{a}(1), \ldots, \mathfrak{a}(\mathrm{k}), \quad \mathfrak{b}(1), \ldots, \mathfrak{b}(\ell-1), \quad \mathfrak{b}(\ell)<m, \quad m, \ldots, m) \text { and hence } \\
& \mathfrak{q}^{+}=(\mathfrak{a}(1), \ldots, \mathfrak{a}(\mathbf{k}), \quad \mathfrak{b}(1), \ldots, \mathfrak{b}(\ell-1), \quad \mathfrak{b}(\ell)+1, \quad 1, \ldots, 1),
\end{aligned}
$$

then Spoiler uses the increment gadget $\mathrm{INC}_{\ell}^{\text {right }}$ to reach $\mathfrak{q}^{+}$on the output from $\mathfrak{q}$ on the input. If

$$
\begin{aligned}
& \mathfrak{q}=(\mathfrak{a}(1), \ldots, \mathfrak{a}(\ell-1), \quad \mathfrak{a}(\ell)<n, \quad n, \ldots, n, \quad m, \ldots, m) \text { and hence } \\
& \mathfrak{q}^{+}=(\mathfrak{a}(1), \ldots, \mathfrak{a}(\ell-1), \quad \mathfrak{a}(\ell)+1, \quad 1, \ldots, 1, \quad 1, \ldots, 1),
\end{aligned}
$$

then Spoiler uses $\mathrm{INC}_{\ell}^{\text {left }}$. Thus, for every valid configuration $\mathfrak{q}$ with $\alpha(\mathfrak{q})<$ $n^{\mathrm{k}} m^{\mathrm{k}}-1$ there is exactly one applicable increment gadget. The increment gadgets
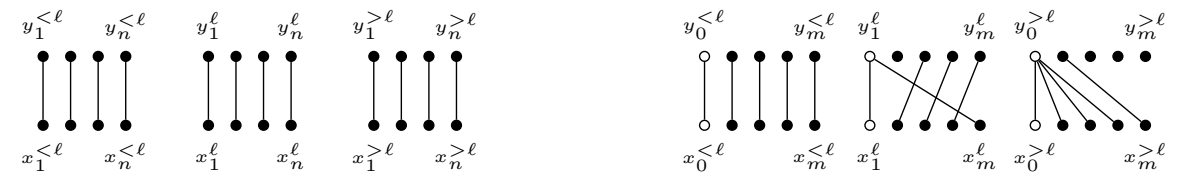

$$
\mathrm{INC}_{\ell}^{\text {right }} \subseteq \mathrm{A}_{n}
$$

$$
\mathrm{INC}_{\ell}^{\mathrm{right}} \subseteq \mathrm{B}_{m}
$$
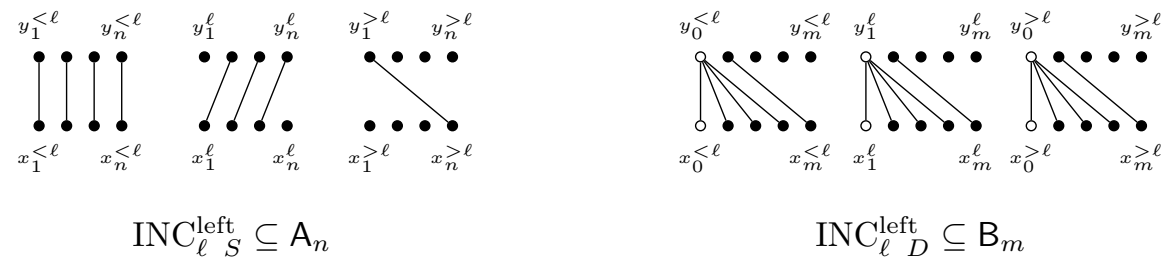

$\mathrm{INC}_{\ell}^{\mathrm{left}} \subseteq \mathrm{B}_{m}$

Fig. 4. The increment gadgets.

are shown in Figure 4. All input vertices $x_{j}^{i}$ have at most one output vertex $y_{j^{\prime}}^{i}$ 
as neighbor. Furthermore, if the gadget is applicable to a valid configuration $\mathfrak{q}=(\mathfrak{a}, \mathfrak{b}, \emptyset)$, then the unique neighbor of $x_{\mathfrak{a}(i)}^{i}$ is $y_{\mathfrak{a}^{+}(i)}^{i}$ and the unique neighbor of $x_{\mathfrak{b}(i)}^{i}$ is $y_{\mathfrak{b}^{+}(i)}^{i}$. This enables Spoiler to reach $\mathfrak{q}^{+}$on the output from $\mathfrak{q}$ on the input by the following procedure. First, Spoiler places the remaining pebble on $y_{\mathfrak{a}^{+}(1)}^{1}$. Since this vertex is adjacent to $x_{\mathfrak{a}(1)}^{1}$, Duplicator has to answer with $y_{\mathfrak{b}^{+}(1)}^{1}$, the only vertex that is adjacent to $x_{\mathfrak{b}(1)}^{1}$. Afterwards, Spoiler picks up the pebble pair from $\left(x_{\mathfrak{a}(1)}^{1}, x_{\mathfrak{b}(1)}^{1}\right)$. On the second block Spoiler proceeds the same way: he pebbles $y_{\mathfrak{a}^{+}(2)}^{2}$, forces the position $\left(y_{\mathfrak{a}^{+}(2)}^{2}, y_{\mathfrak{b}^{+}(2)}^{2}\right)$ and picks up the pebbles from $\left(x_{\mathfrak{a}(2)}^{2}, x_{\mathfrak{b}(2)}^{2}\right)$. By iterating this procedure Spoiler reaches $\mathfrak{q}^{+}$on the output.

If Spoiler tries to move a configuration through one increment gadget that is not applicable, then Duplicator can answer with an invalid configuration on the output as follows. On the one hand, if the gadget is not applicable because some $\mathfrak{b}(i)$ does not have the specified value, then $x_{\mathfrak{b}(i)}^{i}$ is adjacent to $y_{0}^{i}$. On the other hand, if some $\mathfrak{a}(i)$ has the wrong value, then $x_{\mathfrak{a}(i)}^{i}$ is not adjacent to an output vertex. In both cases Duplicator can safely pebble $y_{0}^{i}$ if Spoiler queries some $y_{j}^{i}$ and hence maintain an invalid output position. The next lemma provides these strategies, a formal proof is given in the full version of the paper.

Lemma 5. Let $\mathfrak{q}=(\mathfrak{a}, \mathfrak{b}, T)$ be a configuration and INC an increment gadget.

1. If INC is applicable to $\mathfrak{q}$, then Spoiler can reach $\mathfrak{q}^{+}$on the output from $\mathfrak{q}$ on the input of INC.

2. If INC is applicable to $\mathfrak{q}$, then there is a winning strategy for Duplicator with boundary function $h_{\mathfrak{q}}^{x}$ on the input and $h_{\mathfrak{q}^{+}}^{y}$ on the output.

3. If INC is not applicable to $\mathfrak{q}$, then there is a winning strategy for Duplicator with boundary function $h_{\mathfrak{q}}^{x}$ on the input and $h_{\mathfrak{q}_{\mathrm{inv}}}^{y}$ on the output for an invalid configuration $\mathfrak{q}_{\mathrm{inv}}$.

The switch is an extension of the "multiple input one-way switch" defined in [3] (which in turn is a generalization of [11]). The difference is that the old switch can only be used for the case $n=1$. It requires some work to adjust the old switch to make it work for the more general setting. The technical details of the switch are shifted to Appendix A.2. At this point we focus on a high level description of the strategies and formalize them in Lemma 6. The proof of this lemma can also be found in Appendix A.2.

As mentioned earlier, Spoiler can simply move a valid position from the input to the output of the switch (Lemma 6(i)). Duplicator has a winning strategy called output strategy, where any position is on the output and $h_{\mathbf{0}}^{x}$ is on the input (Lemma 6(ii)). This ensures that Spoiler cannot move backwards to reach $\mathfrak{q}$ on the input from $\mathfrak{q}$ on the output. Hence, this strategy forces Spoiler to play through the switches in the intended direction (as indicated by arrows Figure22). Furthermore, for every invalid $\mathfrak{q}_{\text {inv }}$ Duplicator has a winning strategy where $h_{\mathfrak{q}_{\text {inv }}}^{x}$ is on the input and $h_{\mathbf{0}}^{y}$ is on the output (Lemma 6(iii)), which ensures that Spoiler cannot move invalid positions through the switch. This strategy is used by Duplicator whenever Spoiler plays on an increment gadget that is 
not applicable. By Lemma 5. Duplicator can force an invalid configuration on the output of that increment gadget and hence on the input of the subsequent switch.

To ensure that Spoiler picks up all pebbles when reaching $\mathfrak{q}$ on the output from $\mathfrak{q}$ on the input, Duplicator has a critical input strategy with $\mathfrak{q}$ on the input and $h_{0}^{y}$ on the output (Lemma 6(iv)). The critical positions are either contained in an output strategy, where $\mathfrak{q}$ is on the output, or (for technical reasons) in a restart strategy. If Duplicator plays according to this input strategy, the only way for Spoiler to bring $\mathfrak{q}$ from the input to the output is to pebble an output critical position inside the switch (using all the pebbles) and force Duplicator to switch to the corresponding output strategy.

Lemma 6. For every configuration $\mathfrak{q}=(\mathfrak{a}, \mathfrak{b}, T)$, the following statements hold in the existential $(\mathrm{k}+1)$-pebble game on the switch:

(i) If $\mathfrak{q}$ is valid, then Spoiler can reach $\mathfrak{q}$ on the output from $\mathfrak{q}$ on the input.

(ii) Duplicator has a winning strategy $\mathcal{H}_{\mathfrak{q}}^{\text {out }}$ with boundary function $h_{\mathbf{0}}^{x} \cup h_{\mathfrak{q}}^{y}$.

(iii) If $\mathfrak{q}$ is invalid, then Duplicator has a winning strategy $\mathcal{H}_{\mathfrak{q}}^{\text {restart }}$ with boundary function $h_{\mathfrak{q}}^{x} \cup h_{\mathbf{0}}^{y}$.

(iv) If $\mathfrak{q}$ is valid, then Duplicator has a critical strategy $\mathcal{H}_{\mathfrak{q}}^{\text {in }}$ with boundary function $h_{\mathfrak{q}}^{x} \cup h_{\mathbf{0}}^{y}$ and sets of restart critical positions $\mathcal{C}_{\mathfrak{q}, t}^{\text {restart-crit }}$ (for $t \in[\mathrm{k}]$ ) and output critical positions $\mathcal{C}_{\mathfrak{q}}^{\text {out-crit }}$ such that:

(a) $\operatorname{crit}\left(\mathcal{H}_{\mathfrak{q}}^{\text {in }}\right)=\bigcup_{t \in[\mathrm{k}]} \mathcal{C}_{\mathfrak{q}, t}^{\text {restart-crit }} \cup \mathcal{C}_{\mathfrak{q}}^{\text {out-crit }}$,

(b) $\mathcal{C}_{\mathfrak{q}, t}^{\text {restart-crit }} \subseteq \mathcal{H}_{(\mathfrak{a}, \mathfrak{b},\{t\})}^{\text {restart }}$ and

(c) $\mathcal{C}_{\mathfrak{q}}^{\text {out-crit }} \subseteq \mathcal{H}_{\mathfrak{q}}^{\text {out }}$.

At the beginning of the game we want that Spoiler can reach the start configuration $\alpha^{-1}(0)$ on $\mathrm{x}$, which is the pebble position $\left\{\left(\mathrm{x}_{1}^{1}, \mathrm{x}_{1}^{1}\right), \ldots,\left(\mathrm{x}_{1}^{k}, \mathrm{x}_{1}^{k}\right)\right\}$. To ensure this, we use the initialization gadget and identify its output vertices $y_{j}^{i}$ with the block of $x_{j}^{i}$ vertices. As for the switch, this gadget is an extension of the initialization gadget presented in [3]. We now describe the strategies on the initialization gadget and formalize them in Lemma 7. A detailed description of the gadget and a proof of Lemma 7 is given in Appendix A.3. The main property of the gadget is that Spoiler can reach the start position $\mathfrak{q}$ at the boundary (i) and Duplicator has a corresponding counter strategy (ii) in this situation. Furthermore, if an arbitrary position occurs at the boundary during the game, Duplicator has a strategy to survive (iii). This is only a critical strategy, but Duplicator can switch to the initial strategy (hence "restart" the game) if Spoiler moves to one of the critical positions.

Lemma 7. Let $\mathfrak{q}=\alpha^{-1}(0)$. The following holds in the existential $(\mathrm{k}+1)$-pebble game on INIT:

(i) Spoiler can reach $\mathfrak{q}$ on the output.

(ii) There is a winning strategy $\mathcal{I}^{\text {init }}$ for Duplicator with boundary function $h_{\mathfrak{q}}^{y}$.

(iii) For every (valid or invalid) configuration $\mathfrak{q}^{\prime}$ there is a critical strategy $\mathcal{I}_{\mathfrak{q}^{\prime}}^{\text {init }}$ with boundary function $h_{\mathfrak{q}^{\prime}}^{y}$ and $\operatorname{crit}\left(\mathcal{I}_{\mathfrak{q}^{\prime}}^{\text {init }}\right) \subseteq \mathcal{I}^{\text {init }}$. 


\subsection{Proof of Theorem 1}

The size of the vertex set in every gadget is linear in $n$ on Spoiler's side and linear in $m$ on Duplicator's side. Since the overall construction uses a constant number of gadgets it follows that $\left|V\left(\mathrm{~A}_{n}\right)\right|=O(n)$ and $\left|V\left(\mathrm{~B}_{m}\right)\right|=O(m)$. To prove the lower bound on the number of rounds Spoiler needs to win the existential $(k+1)$ pebble game we provide a sequence of critical strategies in Lemma 8 satisfying the properties stated in Lemma 4 . For a critical strategy $\mathcal{S}$ we let $\widehat{\mathcal{S}}:=\mathcal{S} \backslash \operatorname{crit}(\mathcal{S})$.

Lemma 8. Spoiler has a winning strategy in the existential $(\mathrm{k}+1)$-pebble game on $\mathrm{A}_{n}$ and $\mathrm{B}_{m}$. Furthermore, there is a sequence of critical strategies for Duplicator $\mathcal{G}^{\text {start }}, \mathcal{F}_{1}, \mathcal{G}_{1}, \mathcal{F}_{2}, \mathcal{G}_{2}, \ldots, \mathcal{G}_{n^{k} m^{k}-2}, \mathcal{F}_{n^{k} m^{k}-1}$ such that

$$
\begin{aligned}
\operatorname{crit}\left(\mathcal{G}^{\text {start }}\right) & \subseteq \widehat{\mathcal{F}}_{1}, & & \\
\operatorname{crit}\left(\mathcal{G}_{i}\right) & \subseteq \widehat{\mathcal{F}}_{i+1} \cup \widehat{\mathcal{G}}^{\text {start }}, & & 1 \leq i \leq n^{\mathrm{k}} m^{\mathrm{k}}-2, \\
\operatorname{crit}\left(\mathcal{F}_{i}\right) & \subseteq \widehat{\mathcal{G}}_{i} \cup \widehat{\mathcal{G}}^{\text {start }}, & & 1 \leq i \leq n^{\mathrm{k}} m^{\mathrm{k}}-2 .
\end{aligned}
$$

Proof (Proof of Theorem 11). For $k=2$ the theorem follows from [4]. For $k \geq 3$ consider the structures $\mathrm{A}_{n}$ and $\mathrm{B}_{m}$ (for $\mathrm{k}=k-1$ ) defined above. By Lemma 8 Spoiler wins the existential $k$-pebble game on $\mathrm{A}_{n}$ and $\mathrm{B}_{m}$. Furthermore, it follows via Lemma 4 that Spoiler needs at least $\Omega\left(n^{k-1} m^{k-1}\right)$ rounds to win the game. To get structures with exactly $n$ and $m$ vertices we take the largest $n^{\prime}, m^{\prime}$ such that $\left|V\left(\mathrm{~A}_{n^{\prime}}\right)\right| \leq n,\left|V\left(\mathrm{~B}_{m^{\prime}}\right)\right| \leq m$ and fill up the structures with an appropriate number of isolated vertices.

Proof (Proof of Lemma 8). To show that Spoiler has a winning strategy it suffices to prove the following three statements:

(1) Spoiler can reach the position $\alpha^{-1}(0)$ on $\times$ from $\emptyset$,

(2) Spoiler can reach $\alpha^{-1}(i+1)$ on $\mathrm{x}$ from $\alpha^{-1}(i)$ on $\mathrm{x}\left(\right.$ for $\left.i<n^{\mathrm{k}} m^{\mathrm{k}}-1\right)$ and

(3) Spoiler wins from $\alpha^{-1}\left(n^{\mathrm{k}} m^{\mathrm{k}}-1\right)$ on $\mathrm{x}$.

Assertion (1) follows from Lemma 7 and (3) is ensured by the winning gadget. For (2), Spoiler starts with the position $\mathfrak{q}=\alpha^{-1}(i)$ on $\mathrm{x}$. Since $i<n^{\mathrm{k}} m^{\mathrm{k}}-1$ there is exactly one increment gadget applicable to $\mathfrak{q}$. Spoiler uses Lemma 5 to reach $\mathfrak{q}^{+}=\alpha^{-1}(i+1)$ on the output of that gadget. By applying Lemma 6.(i) twice, Spoiler can pebble $\mathfrak{q}^{+}$through the two switches to the $x$ vertices.

To define the sequence of global critical strategies we combine the partial critical strategies on the gadgets using the $\uplus$-operator. There are three types of strategies: $\mathcal{G}^{\text {start }}, \mathcal{F}_{i}$ and $\mathcal{G}_{i}$. To define $\mathcal{G}_{i}$ we let $\mathfrak{q}=\alpha^{-1}(i)$. Duplicator plays according to $h_{\mathfrak{q}}^{\times}$on $\mathrm{x}$ and according to $h_{\mathbf{0}}^{\mathrm{y}}$ on $\mathrm{y}$. She plays according to this strategy in the case when Spoiler reaches "q on $x$ ". The critical strategy $\mathcal{G}_{i}$ is the combination of the following (pairwise connectable) strategies on the gadgets:

- The critical strategy $\mathcal{I}_{\mathfrak{q}}^{\text {init }}$ on the initialization gadget (Lemma 7).

- The winning strategy with boundary $h_{\mathfrak{q}}^{x}$ and $h_{\mathfrak{q}^{+}}^{y}$ on the increment gadget applicable to $\mathfrak{q}$ (Lemma 5 ). 
- The critical input strategy $\mathcal{H}_{\mathfrak{q}^{+}}^{\text {in }}$ on the switch following the applicable increment gadget (Lemma 6).

- The winning strategy with boundary $h_{\mathfrak{q}}^{x}$ and $h_{\mathfrak{q}_{\text {inv }}}^{y}$ on the other increment gadgets not applicable to $\mathfrak{q}$ (Lemma 5).

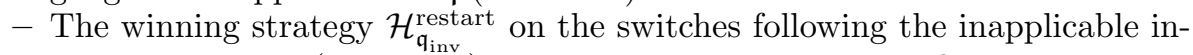
crement gadgets (Lemma 6). Here, $\mathfrak{q}_{\mathrm{inv}}$ is the invalid configuration on the output of the corresponding increment gadget.

- The output winning strategy $\mathcal{H}_{\mathfrak{q}}^{\text {out }}$ on the single switch (Lemma 6).

If in the above setting Spoiler increments $\mathfrak{q}$ through the applicable increment gadget and moves $\mathfrak{q}^{+}=\alpha^{-1}(i+1)$ through the subsequent switch, then Duplicator switches to the strategy $\mathcal{F}_{i+1}$. To define $\mathcal{F}_{i}$ we fix $\mathfrak{q}=\alpha^{-1}(i)$. In this strategy, Duplicator plays according to $h_{\mathbf{0}}^{\times}$on $\mathrm{x}$ and according to $h_{\mathfrak{q}}^{\mathrm{y}}$ on y. This critical strategy is the combination of the following strategies on the gadgets.

- The critical strategy $\mathcal{I}_{0}^{\text {init }}$ on the initialization gadget.

- The winning strategy with boundary $h_{0}^{x}$ and $h_{0}^{y}$ on the increment gadgets.

- The output strategy $\mathcal{H}_{\mathfrak{q}}^{\text {out }}$ on the switches following the increment gadgets.

- The critical input strategy $\mathcal{H}_{\mathfrak{q}}^{\text {in }}$ on the single switch.

The critical positions in the strategies $\mathcal{G}_{i}$ and $\mathcal{F}_{i}$ are inside the switches and the initialization gadget. Recall that by Lemma 6 (iv) the critical positions on the switch can be divided into restart critical positions and output critical positions. Furthermore, all output critical positions of $\mathcal{G}_{i}$, which are inside the switch following the applicable increment gadget, are contained as non-critical positions in $\mathcal{F}_{i+1}$. All output critical position in $\mathcal{F}_{i}$, which are inside the single switch, are contained as non-critical positions in $\mathcal{G}_{i}$. Now we define $\mathcal{G}^{\text {start }}$, which contains all other critical positions of $\mathcal{G}_{i}$ and $\mathcal{F}_{i}$. The critical strategy $\mathcal{G}^{\text {start }}$ is the union of several other global strategies. The first one is $\mathcal{G}^{\text {init }}$, which is defined as $\mathcal{G}_{0}$ except that it contains the winning strategy $\mathcal{I}^{\text {init }}$ on the initialization gadget. Thus, by Lemma 7, it contains every critical position on the initialization gadget as non-critical position. Note that the output critical positions of $\mathcal{G}^{\text {init }}$ are contained as non-critical positions in $\mathcal{F}_{1}$. Since $\mathcal{G}^{\text {init }}$ handles the critical positions on the initialization gadget and we discussed the output critical positions on the switches, it remains to consider the restart critical positions of the strategies. For this we construct a strategy $\mathcal{G}_{i}^{\text {restart }}$ to handle the restart critical positions of $\mathcal{G}_{i}$ (for $i \geq 1$ ) and of $\mathcal{G}^{\text {init }}$ (for $i=0$ ). Furthermore, we define for every $i \geq 1$ a strategy $\mathcal{F}_{i}^{\text {restart }}$ to handle the restart critical positions of $\mathcal{F}_{i}$.

For $0 \leq i \leq n^{\mathrm{k}} m^{\mathrm{k}}-2$ and $t \in[\mathrm{k}]$ we let $\mathfrak{q}=\alpha^{-1}(i)=(\mathfrak{a}, \mathfrak{b}, \emptyset)$ and $\mathfrak{q}_{t}$ be the invalid configuration $(\mathfrak{a}, \mathfrak{b},\{t\})$. The global strategy $\mathcal{G}_{i, t}^{\text {restart }}$ is the combination of the following strategies on the gadgets.

- The critical strategy $\mathcal{I}_{\mathfrak{q}_{t}}^{\text {init }}$ on the initialization gadget.

- The winning strategy with boundary $h_{\mathfrak{q}_{t}}^{x}$ and $h_{\mathfrak{q}_{\text {inv }}}^{y}$ on the increment gadgets. Note that, since $\mathfrak{q}_{t}$ is invalid, no increment gadget is applicable to $\mathfrak{q}_{t}$.

- The winning strategy $\mathcal{H}_{\mathfrak{q}_{\text {inv }}}^{\text {restart }}$ on the switches following the increment gadgets. Again, $\mathfrak{q}_{\text {inv }}$ is the invalid configuration at the output of the preceding increment gadget. 
- The output winning strategy $\mathcal{H}_{\mathfrak{q}_{t}}^{\text {out }}$ on the single switch.

Finally, we let $\mathcal{G}_{i}^{\text {restart }}:=\bigcup_{i \in[\mathrm{k}]} \mathcal{G}_{i, t}^{\text {restart }}$. Note that by Lemma 6, (iv) every restart critical position of $\mathcal{G}_{i}$ is contained in $\mathcal{G}_{i}^{\text {restart }}$ and every restart critical position of $\mathcal{G}^{\text {init }}$ is contained in $\mathcal{G}_{0}^{\text {restart }}$. Now we define for $1 \leq i \leq n^{\mathrm{k}} m^{\mathrm{k}}-2, t \in[\mathrm{k}]$, $\mathfrak{q}=\alpha^{-1}(i)=(\mathfrak{a}, \mathfrak{b}, \emptyset)$ and $\mathfrak{q}_{t}:=(\mathfrak{a}, \mathfrak{b},\{t\})$ the strategy $\mathcal{F}_{i, t}^{\text {restart }}$ analogously. It consists of the following partial strategies.

- The critical strategy $\mathcal{I}_{\mathbf{0}}^{\text {init }}$ on the initialization gadget.

- The winning strategy with boundary $h_{0}^{x}$ and $h_{\mathbf{0}}^{y}$ on the increment gadgets.

- The winning strategy $\mathcal{H}_{0}^{\text {restart }}$ on the switches after the increment gadgets.

- The winning strategy $\mathcal{H}_{\mathfrak{q}_{t}}^{\text {restart }}$ on the single switch.

In the end we let $\mathcal{F}_{i}^{\text {restart }}$ be the union of all $\mathcal{F}_{i, t}^{\text {restart }}$. Note that every restart critical position of $\mathcal{F}_{i}$ is contained as non-critical position in $\mathcal{F}_{i}^{\text {restart }}$. Finally, let

$$
\mathcal{G}^{\text {start }}:=\mathcal{G}^{\text {init }} \cup \bigcup_{0 \leq i \leq n^{k} m^{k}-2} \mathcal{G}_{i}^{\text {restart }} \cup \bigcup_{1 \leq i \leq n^{k} m^{k}-2} \mathcal{F}_{i}^{\text {restart }} .
$$

To conclude the proof note that the critical positions of $\mathcal{G}_{i}^{\text {restart }}$ and $\mathcal{F}_{i}^{\text {restart }}$ are inside the initialization gadget and hence contained in $\widehat{\mathcal{G}}^{\text {init }}$. Thus they are not critical positions of $\mathcal{G}^{\text {start }}$. Hence, $\operatorname{crit}\left(\mathcal{G}^{\text {start }}\right)=\operatorname{crit}\left(\mathcal{G}^{\text {init }}\right) \subseteq \widehat{\mathcal{F}}_{1}$.

\section{Conclusion}

We have proven an optimal lower bound of $\Omega\left(n^{k-1} d^{k-1}\right)$ on the number of nested propagation steps in the $k$-consistency procedure on constraint networks with $n$ variables and domain size $d$. It follows that every parallel propagation algorithm has to perform at least $\Omega\left(n^{k-1} d^{k-1}\right)$ sequential steps. Using $(n+d)^{O(k)}$ processors (one for every instance of the inference rule), $k$-consistency can be computed in $O\left(n^{k-1} d^{k-1}\right)$ parallel time, which is optimal for propagation algorithms. In addition, the best sequential algorithm runs in $O\left(n^{k} d^{k}\right)$. The overhead compared to the parallel approach is mainly caused by the time needed to search for the next inconsistent assignment that might be propagated - and this seems to be the only task that can be parallelized.

Although we have proven an optimal lower bound in the general setting, it might be interesting to investigate the propagation depth of $k$-consistency on restricted classes of structures. Especially, if in such cases the propagation depth is bounded by $O(\log (n+d))$, we know that $k$-consistency is in NC and hence parallelizable. 


\section{References}

1. Atserias, A., Kolaitis, P., Vardi, M.: Constraint propagation as a proof system. In: Wallace, M. (ed.) Principles and Practice of Constraint Programming CP 2004. Lecture Notes in Computer Science, vol. 3258, pp. 77-91. Springer Berlin Heidelberg (2004)

2. Berkholz, C.: Lower bounds for existential pebble games and k-consistency tests. In: Logic in Computer Science (LICS), 2012 27th Annual IEEE Symposium on. pp. 25-34 (2012)

3. Berkholz, C.: Lower bounds for existential pebble games and k-consistency tests. Logical Methods in Computer Science 9(4) (2013), http://arxiv.org/abs/1205. 0679

4. Berkholz, C., Verbitsky, O.: On the speed of constraint propagation and the time complexity of arc consistency testing. In: Chatterjee, K., Sgall, J. (eds.) Mathematical Foundations of Computer Science 2013, Lecture Notes in Computer Science, vol. 8087, pp. 159-170. Springer Berlin Heidelberg (2013)

5. Cooper, M.C.: An optimal k-consistency algorithm. Artificial Intelligence 41(1), 89 - 95 (1989)

6. Dechter, R., Pearl, J.: A problem simplification approach that generates heuristics for constraint-satisfaction problems. Tech. rep., Cognitive Systems Laboratory, Computer Science Department, University of California, Los Angeles (1985)

7. Feder, T., Vardi, M.Y.: The computational structure of monotone monadic snp and constraint satisfaction: A study through datalog and group theory. SIAM Journal on Computing 28(1), 57-104 (1998)

8. Freuder, E.C.: Synthesizing constraint expressions. Commun. ACM 21, 958-966 (Nov 1978)

9. Gaspers, S., Szeider, S.: The parameterized complexity of local consistency. In: Proc. CP'11. pp. 302-316 (2011)

10. Kasif, S.: On the parallel complexity of discrete relaxation in constraint satisfaction networks. Artificial Intelligence 45(3), 275 - 286 (1990)

11. Kolaitis, P.G., Panttaja, J.: On the complexity of existential pebble games. In: Proc. CSL'03. pp. 314-329 (2003)

12. Kolaitis, P.G., Vardi, M.Y.: On the expressive power of datalog: Tools and a case study. J. Comput. Syst. Sci. 51(1), 110-134 (1995)

13. Kolaitis, P.G., Vardi, M.Y.: A game-theoretic approach to constraint satisfaction. In: Proc AAAI/IAAI'00. pp. 175-181 (2000)

14. Ladkin, P.B., Maddux, R.D.: On binary constraint problems. J. ACM 41(3), 435469 (May 1994), http://doi.acm.org/10.1145/176584.176585

15. Samal, A., Henderson, T.: Parallel consistent labeling algorithms. International Journal of Parallel Programming 16, 341-364 (1987)

16. Susswein, S., Henderson, T., Zachary, J., Hansen, C., Hinker, P., Marsden, G.: Parallel path consistency. International Journal of Parallel Programming 20(6), 453-473 (1991), http://dx.doi.org/10.1007/BF01547895 


\section{A Appendix}

This appendix contains proofs skipped in the main text. We first give a simple proof of the correspondence between the existential pebble game and CSPrefutations (Subsection A.1) followed by the definition and strategies on the switch (Subsection A.2) and the initialization gadget (Subsection A.3).

\section{A.1 Proof of Lemma 2}

Lemma 9. (Reminder of Lemma 2) Let A and B be two relational structures. There is a CSP-refutation for $\mathrm{A}$ and $\mathrm{B}$ of width $k-1$ and depth $d$ if and only if Spoiler has a strategy to win the existential k-pebble game on $\mathrm{A}$ and $\mathrm{B}$ within d rounds.

Proof. For one direction assume that there is a CSP-refutation $P$ of depth $d$ and width $k-1$. We show by induction over the depth that every partial mapping $p$ of depth $i$ occurring in the refutation defines a position of pebbles from which Spoiler can win the existential $k$-pebble game within $i$ rounds. It follows that Spoiler can win the game from $\emptyset$ (all pebbles off the board) within $d$ rounds. All mappings of depth $i=0$ are axioms and thus not partial homomorphisms. Hence, Spoiler wins immediately. For the induction step assume that $p$ has depth $i>0$. Therefore, $|p|<k$ and $p$ is derived from $p_{1}^{\prime} \cup\left\{x \mapsto a_{1}\right\}, \ldots, p_{n}^{\prime} \cup\left\{x \mapsto a_{n}\right\}$ $\left(p_{j}^{\prime} \subseteq p\right)$ each of depth $<i$. Spoiler can now reach one of these positions within one round by placing the remaining pebble on $x$. Depending on Duplicator's choice (some $a_{j} \in V(\mathrm{~B})$ ) Spoiler moves to $p_{j}^{\prime} \cup\left\{x \mapsto a_{j}\right\}$ by picking up the pebbles in $p \backslash p_{j}^{\prime}$. By induction assumption, Spoiler can win from $p_{j}^{\prime} \cup\left\{x \mapsto a_{j}\right\}$ within $<i$ rounds and hence he can win from $p$ within $i$ rounds.

To prove the other direction we show by induction over the number of rounds that if Spoiler has an $i$-round winning strategy from a position $p$, then some $p^{\prime} \subseteq p$ has a CSP-derivation of depth $i$. Since we assume that Spoiler has a $d$-round winning strategy from $\emptyset$, the lemma follows. For $i=0$ the 0 -round winning positions are precisely the axioms in our derivation system. Assume that Spoiler has an $i$-round winning strategy from $p$. In the next round in his strategy Spoiler first has to pick up at least one pebble. Let $p^{\prime} \subseteq p$ be the new position and note that $\left|p^{\prime}\right|<k$. By the definition of the game Spoiler also has a $i$-round winning strategy from $p^{\prime}$. Let $x \in V(\mathrm{~A})$ be the element on which the next pebble is set. Since Spoiler has a strategy to win against every possible choice of Duplicator, we know that $p^{\prime} \cup\left\{x \mapsto a_{1}\right\}, \ldots, p^{\prime} \cup\left\{x \mapsto a_{n}\right\}$ are positions from which Spoiler can win the game within $i-1$ rounds. For all these positions there is a $p_{j} \subseteq p^{\prime} \cup\left\{x \mapsto a_{j}\right\}$ that has a derivation of depth at most $i-1$ by induction assumption. If for some $j$ it holds that $p_{j} \subseteq p^{\prime} \subseteq p$ we are done. Otherwise, all $p_{j}$ are of the form $p_{j}=p_{j}^{\prime} \cup\left\{x \mapsto a_{j}\right\}$ with $p_{j}^{\prime} \subseteq p^{\prime} \subseteq p$. Thus, $p$ has a derivation of depth at most $i$ by applying the derivation rule (1). 


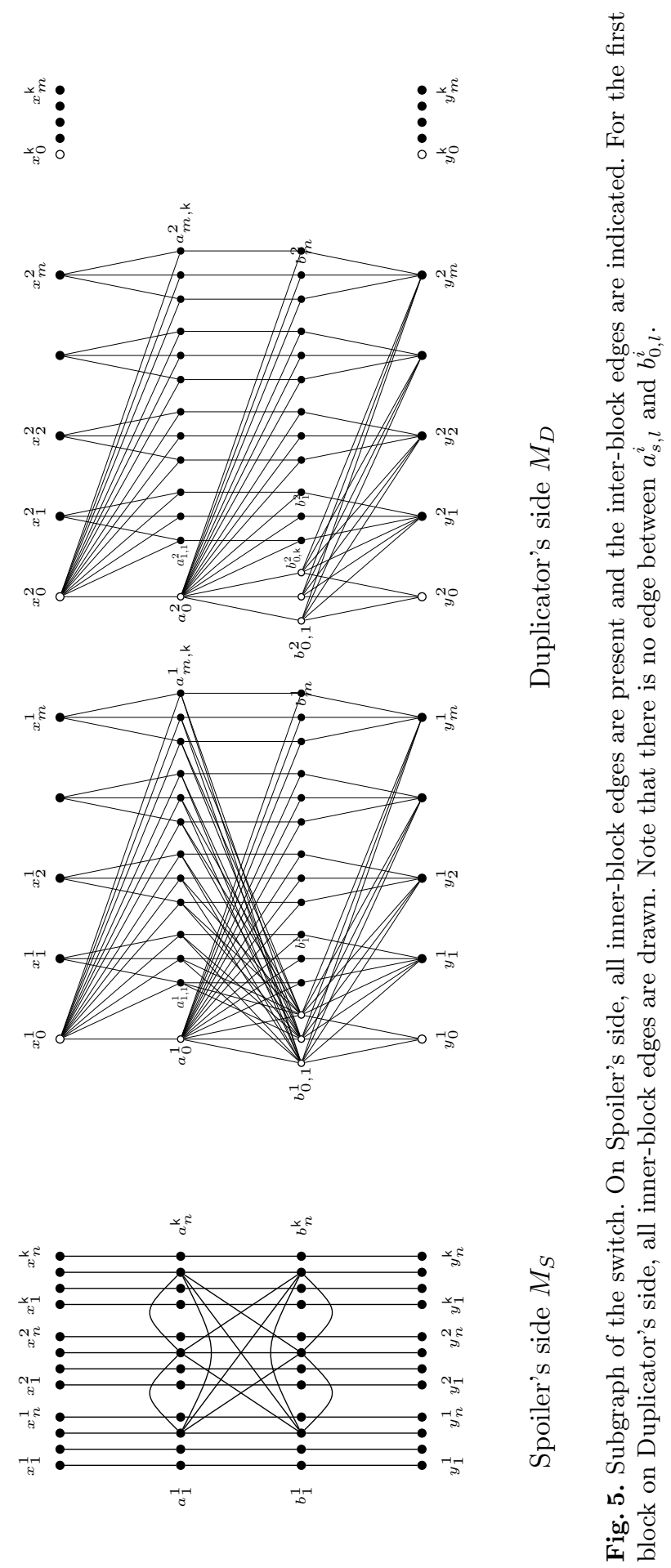

XVIII 


\section{A.2 The Switch}

For the reader familiar with the literature it is worth noting that the switch presented here is an extension of the "multiple input one-way switch" defined in 2[3]. The difference is that the old switch can only be used for the case $n=1$. However, many strategies and technical definitions can directly be extended to this more general setting. The switch in 223] was in turn a further development of the work from Kolaitis and Panttaja [11, who constructed a switch for the special case $n=1$ and $m=2$.

In order to define the switch we construct the two graphs: $M_{S}$ for Spoiler's side and $M_{D}$ for Duplicator's side. Let

$$
\begin{aligned}
V\left(M_{S}\right) & =\left\{x_{j}^{i}, a_{j}^{i}, b_{j}^{i}, y_{j}^{i} \mid i \in[\mathrm{k}], j \in[n]\right\}, \\
E\left(M_{S}\right) & =\left\{\left\{x_{j}^{i}, a_{j}^{i}\right\},\left\{a_{j}^{i}, b_{j}^{i}\right\},\left\{b_{j}^{i}, y_{j}^{i}\right\} \mid i \in[\mathrm{k}], j \in[n]\right\} \\
& \cup\left\{\left\{a_{j}^{i}, a_{j^{\prime}}^{i^{\prime}}\right\},\left\{b_{j}^{i}, b_{j^{\prime}}^{i^{\prime}}\right\},\left\{a_{j}^{i}, b_{j^{\prime}}^{i^{\prime}}\right\} \mid i, i^{\prime} \in[\mathrm{k}] ; i \neq i^{\prime} ; j, j^{\prime} \in[n]\right\}
\end{aligned}
$$

That is, within one block $i \in[\mathrm{k}]$ of $M_{S}$ the vertices $a_{1}^{i}, a_{2}^{i}, \ldots$ are pairwise connected to $b_{1}^{i}, b_{2}^{i}, \ldots$ and between two blocks $i$ and $i^{\prime}$ every vertex $a_{j}^{i}$ and $b_{j}^{i}$ from block $i$ is connected to every vertex $a_{j^{\prime}}^{i^{\prime}}$ and $b_{j^{\prime}}^{i^{\prime}}$ from block $i^{\prime}$. For Duplicator's side of the graph, we define for $i \in[\mathrm{k}]$ :

$$
\begin{array}{ll}
X^{i}=\left\{x_{s}^{i} \mid 0 \leq s \leq m\right\}, & Y^{i}=\left\{y_{s}^{i} \mid 0 \leq s \leq m\right\} \\
A_{+}^{i}=\left\{a_{s, l}^{i} \mid s \in[m], l \in[\mathrm{k}]\right\}, & A^{i}=A_{+}^{i} \cup\left\{a_{0}^{i}\right\} \\
B_{+}^{i}=\left\{b_{s, l}^{i} \mid s \in[m], l \in[\mathrm{k}]\right\}, & B^{i}=B_{+}^{i} \cup\left\{b_{0, l}^{i} \mid l \in[\mathrm{k}]\right\} .
\end{array}
$$

The set of vertices of $M_{D}$ is

$$
V\left(M_{D}\right)=\bigcup_{i \in[\mathrm{k}]}\left(X^{i} \cup A^{i} \cup B^{i} \cup Y^{i}\right) .
$$

The graphs consist of $\mathrm{k}$ blocks, where the $i$-th block contains all vertices with upper index $i$. Furthermore there are four types of variables (drawn in one row in Figure 2 the input vertices $x$, the output vertices $y$, the vertices $a$ and $b$ (with several indices). Every block of every type of vertices gets a unique color. That is, all $x_{j}^{i}\left(y_{j}^{i}, a_{j}^{i}, b_{j}^{i}\right)$ in $M_{S}$ get the same color as the vertices $X^{i}\left(Y^{i}, A^{i}, B^{i}\right.$, resp.) in $M_{D}$. This ensures that Duplicator always has to answer with vertices of the same type in the same block.

Now we describe the edges in $M_{D}$. We first define the inner-block edges $E^{i}$, which are also shown in Figure 2, and then the inter-block edges $E^{i, j}$ :

$$
\begin{aligned}
E^{i}= & \left(\left\{x_{0}^{i}\right\} \times A^{i}\right) \\
& \cup\left\{\left\{x_{s}^{i}, a_{s, l}^{i}\right\} \mid s \in[m] ; l \in[\mathrm{k}]\right\} \\
& \cup\left(\left\{a_{0}^{i}\right\} \times B^{i}\right) \\
& \cup\left\{\left\{a_{s, l}^{i}, b_{s, l}^{i}\right\} \mid s \in[m] ; l \in[\mathrm{k}]\right\}
\end{aligned}
$$




$$
\begin{aligned}
& \cup\left\{\left\{a_{s, l}^{i}, b_{0, l^{\prime}}^{i}\right\} \mid s \in[m] ; l, l^{\prime} \in[\mathrm{k}] ; l \neq l^{\prime}\right\} \\
& \cup\left\{\left\{b_{s, l}^{i}, y_{s}^{i}\right\} \mid s \in[m] ; l \in[\mathrm{k}]\right\} \\
& \cup\left\{\left\{b_{0, l}^{i}, y_{s}^{i}\right\} \mid s \in[m] \cup\{0\} ; l \in[\mathrm{k}]\right\}, \\
E^{i, j}= & \left\{\left\{a_{s, l}^{i}, a_{s^{\prime}, l^{\prime}}^{j}\right\}, \mid s, s^{\prime} \in[m] ; l, l^{\prime} \in[\mathrm{k}] ; l \neq l^{\prime}\right\} \\
& \cup\left\{\left\{b_{s, l}^{i}, b_{s^{\prime}, l^{\prime}}^{j}\right\} \mid s \in[m], s^{\prime} \in[m] \cup\{0\} ; l, l^{\prime} \in[\mathrm{k}] ; l \neq l^{\prime}\right\} \\
& \cup\left\{\left\{b_{0, l}^{i}, b_{0, l^{\prime}}^{j}\right\} \mid l, l^{\prime} \in[\mathrm{k}]\right\} \\
& \cup\left\{\left\{a_{s, l}^{i}, b_{s^{\prime}, l^{\prime}}^{j}\right\} \mid s \in[m] ; s^{\prime} \in[m] \cup\{0\} ; l, l^{\prime} \in[\mathrm{k}] ; l \neq l^{\prime}\right\} \\
& \cup\left\{\left\{a_{0}^{i}, a_{s, l}^{j}\right\} \mid s \in[m] ; l \in[\mathrm{k}]\right\} \\
& \cup\left\{\left\{a_{0}^{i}, b_{s, l}^{j}\right\} \mid s \in[m] \cup\{0\} ; l \in[\mathrm{k}]\right\}
\end{aligned}
$$

Finally, $E\left(M_{D}\right)=\bigcup_{i \in[\mathrm{k}]} E^{i} \cup \bigcup_{i, j \in[\mathrm{k}] ; i \neq j} E^{i, j}$. The next lemma states the main properties of the switch. For this, recall the definition of critical strategies (Definition 3 on page $\mathrm{V}$. The first statement (i) states that Spoiler can pebble a valid position from the input to the output. Duplicator uses the critical input strategies (iv) to ensure that Spoiler has to pebble a critical position inside the switch while he pebbles the valid position through the switch. Duplicator's output strategy (ii) ensures that Spoiler cannot move backwards (i. e., reach $\mathfrak{q}$ on the input from $\mathfrak{q}$ on the output). The restart strategy (iii) makes sure that Spoiler cannot pebble an invalid position through the switch.

Lemma 10. (Reminder of Lemma 6 ) For every configuration $\mathfrak{q}=(\mathfrak{a}, \mathfrak{b}, T)$, the following statements hold in the existential $(\mathrm{k}+1)$-pebble game on the switch:

(i) If $\mathfrak{q}$ is valid, then Spoiler can reach $\mathfrak{q}$ on the output from $\mathfrak{q}$ on the input.

(ii) Duplicator has a winning strategy $\mathcal{H}_{\mathfrak{q}}^{\text {out }}$ with boundary function $h_{\mathbf{0}}^{x} \cup h_{\mathfrak{q}}^{y}$.

(iii) If $\mathfrak{q}$ is invalid, then Duplicator has a winning strategy $\mathcal{H}_{\mathfrak{q}}^{\text {restart }}$ with boundary function $h_{\mathfrak{q}}^{x} \cup h_{\mathbf{0}}^{y}$.

(iv) If $\mathfrak{q}$ is valid, then Duplicator has a critical strategy $\mathcal{H}_{\mathfrak{q}}^{\text {in }}$ with boundary function $h_{\mathfrak{q}}^{x} \cup h_{\mathbf{0}}^{y}$ and sets of restart critical positions $\mathcal{C}_{\mathfrak{q}, t}^{\text {restart-crit }}$ (for $t \in[\mathrm{k}]$ ) and output critical positions $\mathcal{C}_{\mathfrak{q}}^{\text {out-crit }}$ such that:

(a) $\operatorname{crit}\left(\mathcal{H}_{\mathfrak{q}}^{\text {in }}\right)=\bigcup_{t \in[\mathrm{k}]} \mathcal{C}_{\mathfrak{q}, t}^{\text {restart-crit }} \cup \mathcal{C}_{\mathfrak{q}}^{\text {out-crit }}$,

(b) $\mathcal{C}_{\mathfrak{q}, t}^{\text {restart-crit }} \subseteq \mathcal{H}_{(\mathfrak{a}, \mathfrak{b},\{t\})}^{\text {restart }}$ and

(c) $\mathcal{C}_{\mathfrak{q}}^{\text {out-crit }} \subseteq \mathcal{H}_{\mathfrak{q}}^{\text {out }}$.

Proof. Let $\mathfrak{q}=(\mathfrak{a}, \mathfrak{b}, T)$ be an arbitrary configuration. We first construct the strategy for Spoiler to prove (i). Starting from position $\left\{\left(x_{\mathfrak{a}(1)}^{1}, x_{\mathfrak{b}(1)}^{1}\right), \ldots,\left(x_{\mathfrak{a}(\mathbf{k})}^{\mathbf{k}}, x_{\mathfrak{b}(\mathbf{k})}^{\mathbf{k}}\right)\right\}$, Spoiler places the $(\mathrm{k}+1)$ st pebble on $a_{\mathfrak{a}(1)}^{1}$. Duplicator has to answer with $a_{\mathfrak{b}(1), l_{1}}^{1}$ for some $l_{1} \in[\mathrm{k}]$, mapping the edge $\left\{x_{\mathfrak{a}(1)}^{1}, a_{\mathfrak{a}(1)}^{1}\right\}$ to some edge in (E2). Next, Spoiler picks up the pebble from $x_{\mathfrak{a}(1)}^{1}$ and puts it on $a_{\mathfrak{a}(2)}^{2}$. Again, Duplicator has to answer with $a_{\mathfrak{b}(2), l_{2}}^{2}$ for some $l_{2} \in[\mathrm{k}] \backslash\left\{l_{1}\right\}$. The index $l_{2}$ has to be different from $l_{1}$ because there is an edge between $a_{\mathfrak{a}(1)}^{1}$ and $a_{\mathfrak{a}(2)}^{2}$, but none between $a_{\mathfrak{b}(1), l_{1}}^{1}$ and $a_{\mathfrak{b}(2), l_{1}}^{2}$ in (E8). Following that scheme, Spoiler 
can reach the position $\left\{\left(a_{\mathfrak{a}(1)}^{1}, a_{\mathfrak{b}(1), l_{1}}^{1}\right), \ldots,\left(a_{\mathfrak{a}(\mathbf{k})}^{\mathbf{k}}, a_{\mathfrak{b}(\mathbf{k}), l_{\mathfrak{k}}}^{\mathbf{k}}\right)\right\}$ for pairwise distinct $l_{1}, l_{2}, \cdots, l_{\mathrm{k}}$. Now, Spoiler pebbles $b_{\mathfrak{a}(1)}^{1}$ with the free pebble and Duplicator has to answer with a vertex in $B^{1}$ (due to the vertex-colors) that is adjacent to all $a_{\mathfrak{b}(1), l_{1}}^{1}, \ldots, a_{\mathfrak{b}(\mathrm{k}), l_{\mathfrak{k}}}^{\mathrm{k}}$. This is only the case for $b_{\mathfrak{b}(1), l_{1}}^{1}$ (due to (E4) and (E11)), since every vertex of the form $b_{0, l_{i}}^{1}$ is not adjacent to the vertex $a_{\mathfrak{b}(i), l_{i}}^{i}$ according to (E5) and (E11). Furthermore, $b_{\mathfrak{b}(1), l_{1}}^{1}$ is the only vertex of the form $b_{s, l}^{1}$ (for $s>0$ ) that is adjacent to $a_{\mathfrak{b}(i), l_{i}}^{i}$. In the next step Spoiler picks up the pebble from $a_{\mathfrak{a}(1)}^{1}$ and puts it on $b_{\mathfrak{a}(2)}^{2}$. Duplicator has to answer with a vertex that is adjacent to all vertices $b_{\mathfrak{b}(1), l_{1}}^{1}, a_{\mathfrak{b}(2), l_{2}}^{2}, \ldots, a_{\mathfrak{b}(\mathrm{k}), l_{\mathfrak{k}}}^{\mathrm{k}}$. Because of the missing edges in (E5), (E11) and (E9) (!) the only vertex with this property is $b_{\mathfrak{b}(2), l_{2}}^{2}$. Again, Spoiler picks up the pebble from $a_{\mathfrak{a}(2)}^{2}$ and puts it on $b_{\mathfrak{a}(3)}^{3}$. By the same argument as before, Duplicator has to answer with $b_{\mathfrak{b}(3), l_{3}}^{3}$, which is the only vertex adjacent to all of $b_{\mathfrak{b}(1), l_{1}}^{1}, b_{\mathfrak{b}(2), l_{2}}^{2}, a_{\mathfrak{b}(3), l_{3}}^{3}, \ldots, a_{\mathfrak{b}(\mathbf{k}), l_{\mathfrak{k}}}^{\mathbf{k}}$. Thus, Spoiler can reach $\left\{\left(b_{\mathfrak{a}(1)}^{1}, b_{\mathfrak{b}(1), l_{1}}^{1}\right), \ldots,\left(b_{\mathfrak{a}(\mathbf{k})}^{\mathbf{k}}, b_{\mathfrak{b}(\mathbf{k}), l_{k}}^{\mathbf{k}}\right)\right\}$ and from there he reaches $\left\{\left(y_{\mathfrak{a}(1)}^{1}, y_{\mathfrak{b}(1)}^{1}\right), \ldots,\left(y_{\mathfrak{a}(\mathbf{k})}^{\mathbf{k}}, y_{\mathfrak{b}(\mathrm{k})}^{\mathbf{k}}\right)\right\}$ by successively pebbling the edges $\left\{b_{\mathfrak{a}(i)}^{i}, y_{\mathfrak{a}(i)}^{i}\right\}$.

In order to derive the winning strategies for Duplicator in (ii) and (iii) we consider several total homomorphisms from Spoiler's to Duplicator's side. Consider the edges (E1), (E3) and (E7) connecting • vertices with o vertices in one block of Duplicator's side. They can be used by Duplicator to pebble a o vertex when Spoiler moves upwards. This is the crucial ingredient for Duplicator's output strategies (ii). The first homomorphism is used when Spoiler plays the above strategy to get a valid position through the switch and has already taken all his pebbles from the input vertices. If he tries to pebble input vertices again, then Duplicator can move to $x_{0}^{i}$ and plays according to the following homomorphism:

$$
\begin{aligned}
h_{\mathfrak{q}, \sigma}^{\text {out }}\left(x_{j}^{i}\right) & =x_{0}^{i} & & \\
h_{\mathfrak{q}, \sigma}^{\text {out }}\left(a_{\mathfrak{a}(i)}^{i}\right) & =a_{\mathfrak{b}(i), \sigma(i)}^{i} & & h_{\mathfrak{q}, \sigma}^{\text {out }}\left(a_{j}^{i}\right)=a_{0}^{i}, j \neq \mathfrak{a}(i) \\
h_{\mathfrak{q}, \sigma}^{\text {out }}\left(b_{\mathfrak{a}(i)}^{i}\right) & =b_{\mathfrak{b}(i), \sigma(i)}^{i} & h_{\mathfrak{q}, \sigma}^{\text {out }}\left(b_{j}^{i}\right) & =b_{0, \sigma(j)}^{i}, j \neq \mathfrak{a}(i) \\
h_{\mathfrak{q}, \sigma}^{\text {out }}\left(y_{\mathfrak{a}(i)}^{i}\right) & =y_{\mathfrak{b}(i)}^{i} & & h_{\mathfrak{q}, \sigma}^{\text {out }}\left(y_{j}^{i}\right)=y_{0}^{i}, j \neq \mathfrak{a}(i)
\end{aligned}
$$

where $\sigma \in S_{\mathrm{k}}$ is some permutation on [k]. The next homomorphism is used by Duplicator when there is some valid or invalid configuration $\mathfrak{q}$ at the output of the switch.

$$
\begin{aligned}
h_{\mathfrak{q}}^{\text {out }}\left(x_{j}^{i}\right) & =x_{0}^{i} \\
h_{\mathfrak{q}}^{\text {out }}\left(a_{j}^{i}\right) & =a_{0}^{i} \\
h_{\mathfrak{q}}^{\text {out }}\left(b_{j}^{i}\right) & =b_{0, j}^{i} \\
h_{\mathfrak{q}}^{\text {out }}\left(y_{j}^{i}\right) & =h_{\mathfrak{q}}^{y}\left(y_{j}^{i}\right)
\end{aligned}
$$

Since $h_{\mathfrak{q}}^{\text {out }}$ and all $h_{\mathfrak{q}, \sigma}^{\text {out }}$ are total,

$$
\mathcal{H}_{\mathfrak{q}}^{\text {out }}:= \begin{cases}\wp\left(h_{\mathfrak{q}}^{\text {out }}\right), & \mathfrak{q} \text { is invalid, } \\ \wp\left(h_{\mathfrak{q}}^{\text {out }}\right) \cup \bigcup_{\sigma \in S_{\mathfrak{k}}} \wp\left(h_{\mathfrak{q}, \sigma}^{\text {out }}\right), & \text { otherwise }\end{cases}
$$


is a winning strategy for Duplicator satisfying (ii).

If a homomorphism maps all the $a_{\mathfrak{a}(i)}^{i}$ vertices to $A_{+}^{i}$, then it has to map all $b^{i}$ vertices to $B_{+}^{i}$. This is due to the missing edges in (E5), (E11) and has also been used in Spoiler's strategy above. On the other hand, if for at least one $i \in[\mathrm{k}]$ all $a_{j}^{i}$ are mapped to $a_{0}^{i}$, then every $b_{j}^{i}$ can be mapped to $b_{0, l}^{i}$, where $l$ is chosen such that $a_{\mathfrak{b}(j), l}^{j}$ is not in the image of the homomorphism for every $j$. Duplicator benefits from this, because she can now map the $y_{j}^{i}$ vertices arbitrarily using the edges (E7). This behavior is used in the following restart strategies. Note that a homomorphism mapping some $a_{j}^{i}$ to $a_{0}^{i}$ also maps $x_{j}^{i}$ to $x_{0}^{i}$, hence restart strategies require invalid input positions. For invalid $\mathfrak{q}=(\mathfrak{a}, \mathfrak{b}, T)$, let $\mathcal{H}_{\mathfrak{q}}^{\text {restart }}:=\left\{\wp(h) \mid h \in H_{\mathfrak{q}}^{\text {restart }}\right\}$, where $H_{\mathfrak{q}}^{\text {restart }}$ is the set of total homomorphisms $h$ satisfying the constraints $h\left(x_{j}^{i}\right)=h_{\mathfrak{q}}^{x}\left(x_{j}^{i}\right)$ and $h\left(y_{j}^{i}\right)=y_{0}^{i}$. This set clearly satisfies (iii). As an example fix some $t \in T$ and let $g \in H_{\mathfrak{q}}^{\text {restart }}$ be the following homomorphism:

$$
\begin{aligned}
g\left(x_{j}^{i}\right) & =h_{\mathfrak{q}}^{x}\left(x_{j}^{i}\right), \\
g\left(a_{j}^{i}\right) & =a_{\mathfrak{b}(i), i}^{i}, \text { if } j=\mathfrak{a}(i) \text { and } i \notin T, g\left(a_{j}^{i}\right)=a_{0}^{i}, \text { otherwise, } \\
g\left(b_{j}^{i}\right) & =b_{0, t}^{i}, \\
g\left(y_{j}^{i}\right) & =y_{0}^{i} .
\end{aligned}
$$

It remains to consider the critical input strategies (iv). They formalize the following behavior of Duplicator at the time when Spoiler wants to pebble a configuration $\mathfrak{q}$ through the switch as in (i). Fix a valid configuration $\mathfrak{q}=(\mathfrak{a}, \mathfrak{b}, \emptyset)$. If Spoiler pebbles $a_{\mathfrak{a}(i)}^{i}$ or $b_{\mathfrak{a}(i)}^{i}$, Duplicator answers within $A_{+}^{i}$ or $B^{i} \backslash B_{+}^{i}$, respectively. This allows her to answer on the boundary according to the boundary function defined in (iv). However, she may run into trouble when Spoiler places $\mathrm{k}$ pebbles on $a_{\mathfrak{a}(i)}^{i}$ and $b_{\mathfrak{a}(i)}^{i}$ vertices, because they extend to a $(\mathrm{k}+1)$-clique on Spoiler's side, but not on Duplicator's side (on the blocks $A_{+}^{i}$ and $B^{i} \backslash B_{+}^{i}$ ). These positions form the critical positions where Duplicator switches to an output or restart strategy. If all $\mathrm{k}$ pebbles are on $a_{\mathfrak{a}(1)}^{1}, \ldots, a_{\mathfrak{a}(\mathbf{k})}^{\mathbf{k}}$, as in Spoiler's strategy (i), then Duplicator switches to the output strategy (i. e., she plays according to a homomorphism $h_{\mathfrak{q}, \sigma}^{\text {out }}$ ). In all other cases she switches to a restart strategy. For all $\ell \in[\mathrm{k}]$ and permutations $\sigma$ on [k] we define partial homomorphism $h_{\mathfrak{q}, \sigma, \ell}^{\mathrm{in}}$ as follows:

$$
\begin{aligned}
h_{\mathfrak{q}, \sigma, \ell}^{\text {in }}\left(x_{j}^{i}\right) & =h_{\mathfrak{q}}^{x}\left(x_{j}^{i}\right) \\
h_{\mathfrak{q}, \sigma, \ell}^{\text {in }}\left(a_{\mathfrak{a}(i)}^{i}\right) & =a_{\mathfrak{b}(i), \sigma(i)}^{i}, i \neq \sigma^{-1}(\ell) \\
h_{\mathfrak{q}, \sigma, \ell}^{\text {in }}\left(a_{\mathfrak{a}(i)}^{i}\right) & =\text { undefined, } i=\sigma^{-1}(\ell) \\
h_{\mathfrak{q}, \sigma, \ell}^{\text {in }}\left(a_{j}^{i}\right) & =a_{0}^{i}, j \neq \mathfrak{a}(i) \\
h_{\mathfrak{q}, \sigma, \ell}^{\text {in }}\left(b_{j}^{i}\right) & =b_{0, \ell}^{i} \\
h_{\mathfrak{q}, \sigma, \ell}^{\text {in }}\left(y_{j}^{i}\right) & =y_{0}^{i}
\end{aligned}
$$


We need to check that $h_{\mathfrak{q}, \sigma, \ell}^{\text {in }}$ defines a homomorphism from $M_{S} \backslash\left\{a_{\mathfrak{a}(i)}^{\sigma^{-1}(\ell)}\right\}$ to $M_{D}$. For most parts this is easy to verify. The important part is to check that we do not map edges to the missing pairs in the edge sets (E5), (E8) and (E11) where we require that the indices $l$ and $l^{\prime}$ have to be different. The constraints of (E8) are fulfilled because of the permutation $\sigma$. The constraints of (E5) and (E11) are satisfied because we have chosen $\ell$ such that no vertex maps to $a_{s, \ell}^{i}$ for all $i \in[\mathrm{k}]$ and $s \in[m]$. This also shows that the partial homomorphism cannot be extended to a total homomorphism (where $h_{\mathfrak{q}, \sigma, \ell}^{\mathrm{in}}$ is defined on $a_{\mathfrak{a}(i)}^{i}$ for $i=\sigma^{-1}(\ell)$ ). Now we define a partial homomorphism $h_{\mathfrak{q}, \sigma}^{\text {in }}$ for every permutation $\sigma \in S_{\mathrm{k}}$.

$$
\begin{aligned}
h_{\mathfrak{q}, \sigma}^{\text {in }}\left(x_{j}^{i}\right) & =h_{\mathfrak{q}}^{x}\left(x_{j}^{i}\right), \\
h_{\mathfrak{q}, \sigma}^{\text {in }}\left(a_{\mathfrak{a}(i)}^{i}\right) & =a_{\mathfrak{b}(i), \sigma(i)}^{i}, \\
h_{\mathfrak{q}, \sigma}^{\text {in }}\left(a_{j}^{i}\right) & =a_{0}^{i}, j \neq \mathfrak{a}(i), \\
h_{\mathfrak{q}, \sigma}^{\text {in }}\left(b_{j}^{i}\right) & =\text { undefined, } \\
h_{\mathfrak{q}, \sigma}^{\text {in }}\left(y_{j}^{i}\right) & =y_{0}^{i} .
\end{aligned}
$$

Again it is not hard to see that $h_{\mathfrak{q}, \sigma}^{\text {in }}$ defines a partial homomorphism from $M_{S}$ to $M_{D}$. We cannot extend this partial homomorphism to a total homomorphism, because if we map $b_{\mathfrak{a}(i)}^{i}$ to some $b_{0, l}^{i}$ we will map to a missing edge in (E5) or (E11). Otherwise, if we chose some $b_{\mathfrak{b}(i), l}^{i}$, we will map the edge $\left\{b_{\mathfrak{a}(i)}^{i}, y_{\mathfrak{a}(i)}^{i}\right\}$ in $M_{S}$ to the non-edge $\left\{b_{\mathfrak{b}(i), l}^{i}, y_{0}^{i}\right\}$ in $M_{D}$. Duplicator's input strategy is the family of all subsets of all mappings $h_{\mathfrak{q}, \sigma, \ell}^{\text {in }}$ and $h_{\mathfrak{q}, \sigma}^{\text {in }}$. We are ready to define the critical positions. For all $\sigma \in S_{\mathrm{k}}$ let

$$
h_{\mathfrak{q}, \sigma}^{\text {out-crit }}:=\left\{\left(a_{\mathfrak{a}(i)}^{i}, a_{\mathfrak{b}(i), \sigma(i)}^{i}\right) \mid i \in[\mathrm{k}]\right\}
$$

and for all $\sigma \in S_{\mathrm{k}}$ and $t, u \in[\mathrm{k}]$ and $s \in[n]$

$$
h_{\mathfrak{q}, \sigma, t, u, s}^{\text {restart-crit }}:=\left\{\left(a_{\mathfrak{a}(i)}^{i}, a_{\mathfrak{b}(i), \sigma(i)}^{i}\right) \mid i \in[\mathrm{k}] \backslash\{t\}\right\} \cup\left\{\left(b_{s}^{u}, b_{0, \sigma(t)}^{u}\right)\right\} .
$$

Now we can define the sets used in (iv):

$$
\begin{aligned}
\mathcal{H}_{\mathfrak{q}}^{\text {in }} & =\left\{\wp\left(h_{\mathfrak{q}, \sigma}^{\text {in }}\right) \mid \sigma \in S_{\mathrm{k}}\right\} \cup\left\{\wp\left(h_{\mathfrak{q}, \sigma, \ell}^{\text {in }}\right) \mid \sigma \in S_{\mathrm{k}}, \ell \in[\mathrm{k}]\right\}, \\
\mathcal{C}_{\mathfrak{q}}^{\text {out-crit }} & =\left\{h_{\mathfrak{q}, \sigma}^{\text {out-crit }} \mid \sigma \in S_{\mathrm{k}}\right\}, \\
\mathcal{C}_{\mathfrak{q}, t}^{\text {restart-crit }} & =\left\{h_{\mathfrak{q}, \sigma t, t, u, s}^{\text {restart-crit }} \mid \sigma \in S_{\mathrm{k}}, u \in[\mathrm{k}], s \in[n]\right\}, \\
\operatorname{crit}\left(\mathcal{H}_{\mathfrak{q}}^{\text {in }}\right) & =\bigcup_{t \in[\mathrm{k}]} \mathcal{C}_{\mathfrak{q}, t}^{\text {restart-crit }} \cup \mathcal{C}_{\mathfrak{q}}^{\text {out-crit }} .
\end{aligned}
$$

First note that $h_{\mathfrak{q}, \sigma}^{\text {out-crit }} \subset h_{\mathfrak{q}, \sigma}^{\text {in }}$ and $h_{\mathfrak{q}, \sigma, t, u, s}^{\text {restart-crit }} \subset h_{\mathfrak{q}, \sigma, \sigma(t)}^{\text {in }}$. It holds that $\operatorname{crit}\left(\mathcal{H}_{\mathfrak{q}}^{\text {in }}\right) \subseteq$ $\mathcal{H}_{\mathfrak{q}}^{\text {in }}$. It easily follows from the definitions, that $h_{\mathfrak{q}, \sigma}^{\text {out-crit }} \subset h_{\mathfrak{q}, \sigma}^{\text {out }}$. Furthermore, every $h_{\mathfrak{q}, \sigma, t, u, s}^{\text {restart-crit }}$ can be extended to a homomorphism $g \in \mathcal{H}_{(\mathfrak{a}, \mathfrak{b},\{t\})}^{\text {restart }}$ by defining

$$
g\left(x_{j}^{i}\right)=h_{(\mathfrak{a}, \mathfrak{b},\{t\})}^{x}\left(x_{j}^{i}\right)
$$




$$
\begin{aligned}
g\left(a_{\mathfrak{a}(i)}^{i}\right) & =h_{\mathfrak{q}, \sigma, t, u, s}^{\text {restart-crit }}\left(a_{\mathfrak{a}(i)}^{i}\right)=a_{\mathfrak{b}(i), \sigma(i)}^{i}, \text { if } i \neq t, \\
g\left(a_{\mathfrak{a}(t)}^{t}\right) & =a_{0}^{t} \\
g\left(a_{j}^{i}\right) & =a_{0}^{i}, \text { if } j \neq \mathfrak{a}(i), \\
g\left(b_{j}^{i}\right) & =b_{\sigma(t)}^{i}, \\
g\left(y_{j}^{i}\right) & =y_{0}^{i} .
\end{aligned}
$$

This proves statement b) and c) from (iv). It remains to show that $\mathcal{H}_{\mathfrak{q}}^{\text {in }}$ is a critical strategy with critical positions $\operatorname{crit}\left(\mathcal{H}_{\mathfrak{q}}^{\text {in }}\right)$.

Claim. For all $g \in \mathcal{H}_{\mathfrak{q}}^{\text {in }}$ with $|g| \leq \mathrm{k}$, either $g \in \operatorname{crit}\left(\mathcal{H}_{\mathfrak{q}}^{\text {in }}\right)$ or for all $z \in V\left(M_{S}\right)$ there exist an $h \in \mathcal{H}_{\mathfrak{q}}^{\text {in }}$, such that $g \subseteq h$ and $z \in \operatorname{Dom}(h)$.

Proof. As $g$ is a partial homomorphism from $\mathcal{H}_{\mathfrak{q}}^{\text {in }}$ (which only contains subsets of $h_{\mathfrak{q}, \sigma, \ell}^{\text {in }}$ and $h_{\mathfrak{q}, \sigma}^{\text {in }}$ ), we can fix some $\sigma \in S_{\mathrm{k}}$ and $\ell \in[\mathrm{k}]$ such that $g$ is a subset of the following mapping

$$
\begin{array}{rlrl}
x_{\mathfrak{a}(i)}^{i} & \mapsto x_{\mathfrak{b}(i)}^{i}, & x_{j}^{i} & \mapsto x_{0}^{i}, \text { if } j \neq \mathfrak{a}(i), \\
a_{\mathfrak{a}(i)}^{i} & \mapsto a_{\mathfrak{b}(i), \sigma(i)}^{i}, & a_{j}^{i} \mapsto a_{0}^{i}, \text { if } j \neq \mathfrak{a}(i), \\
b_{j}^{i} & \mapsto b_{0, \ell}^{i}, & \\
y_{j}^{i} & \mapsto y_{0}^{i} . & &
\end{array}
$$

Let $B_{S}:=\left\{b_{j}^{i} \mid i \in[\mathrm{k}], j \in[n]\right\} \subseteq V\left(M_{S}\right)$.

Case 1: $\left|\operatorname{Dom}(g) \cap\left\{a_{\mathfrak{a}(i)}^{i} \mid i \in[\mathrm{k}]\right\}\right|=$ k. In this case, $g=h_{\mathfrak{q}, \sigma}^{\text {out-crit }}$ and hence, $g \in \operatorname{crit}\left(\mathcal{H}_{\mathfrak{q}}^{\text {in }}\right)$.

Case 2: $\left|\operatorname{Dom}(g) \cap\left\{a_{\mathfrak{a}(i)}^{i} \mid i \in[\mathrm{k}]\right\}\right|=\mathrm{k}-1$. If $\operatorname{Dom}(g) \cap B_{S} \neq \emptyset$, then $g=h_{\mathfrak{q}, \sigma, \sigma^{-1}(l), u, s}^{\text {restartcrit }}$ for some $u \in[\mathrm{k}]$ and $s \in[n]$. Thus, we can assume that $\operatorname{Dom}(g) \cap B_{S}=\emptyset$ and show for all $z$ that $g$ satisfies the extension property. If $z=a_{j}^{i}$, then $h_{\mathfrak{q}, \sigma}^{\mathrm{in}}$ extends $g$. If $z=x_{j}^{i}, z=b_{j}^{i}$ or $z=y_{j}^{i}$, then $h_{\mathfrak{q}, \sigma, \ell}^{\text {in }}$ extends $g$.

Case 3: $\left|\operatorname{Dom}(g) \cap\left\{a_{\mathfrak{a}(i)}^{i} \mid i \in[\mathrm{k}]\right\}\right| \leq \mathrm{k}-2$. Let $j_{1}$ and $j_{2}$ be two distinct indices such that $a_{\mathfrak{a}\left(j_{1}\right)}^{j_{1}}, a_{\mathfrak{a}\left(j_{2}\right)}^{j_{2}} \notin \operatorname{Dom}(g)$. Furthermore, we can without loss of generality assume that $\sigma\left(j_{1}\right)=\ell$. For $z \neq a_{\mathfrak{a}\left(j_{1}\right)}^{j_{1}}$ the homomorphism $h_{\mathfrak{q}, \sigma, \ell}^{\text {in }}$ extends $g$. If $z=a_{\mathfrak{a}\left(j_{1}\right)}^{j_{1}}$, then $h_{\mathfrak{q}, \sigma^{\prime}, \ell}^{\text {in }}$ extends $g$, where $\sigma^{\prime}:=\{(i, \sigma(i)) \mid i \in$ $\left.[\mathrm{k}] \backslash\left\{j_{1}, j_{2}\right\}\right\} \cup\left\{\left(j_{1}, \sigma\left(j_{2}\right)\right),\left(j_{2}, \sigma\left(j_{1}\right)\right)\right\}$. 


\section{A.3 The Initialization Gadget}
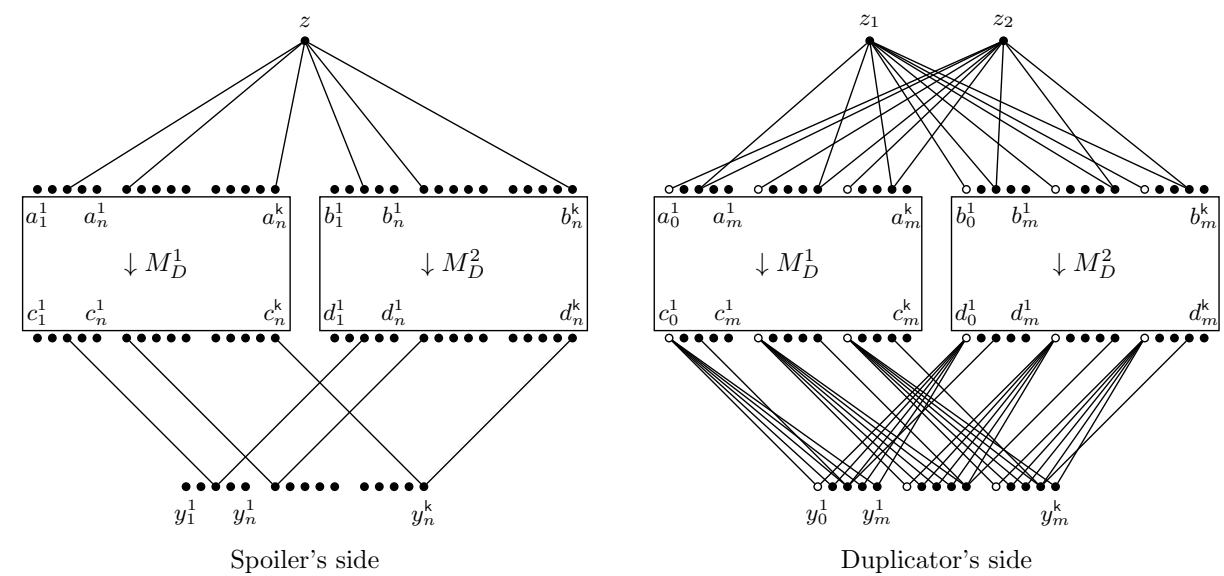

Fig. 6. The initialization gadget (for $\mathrm{k}=3, m=4, n=5, \mathfrak{a}(1)=3, \mathfrak{a}(2)=1, \mathfrak{b}(3)=5$, $\mathfrak{b}(1)=2, \mathfrak{b}(2)=4, \mathfrak{b}(3)=3$.)

At the beginning of the game we want that Spoiler can reach the start configuration $\alpha^{-1}(0)$ on $\mathrm{x}$, which is the pebble position $\left\{\left(\mathrm{x}_{1}^{1}, \mathrm{x}_{1}^{1}\right), \ldots,\left(\mathrm{x}_{1}^{k}, \mathrm{x}_{1}^{k}\right)\right\}$. To ensure this, we introduce an initialization gadget and identify its output vertices $y_{j}^{i}$ with the block of $\mathrm{x}_{j}^{i}$ vertices. The main property is that Spoiler can force the start configuration on the output of the gadget. Another additional property is that from any position on the output of that gadget Duplicator does not lose. This property causes the main difficulties and is needed because other positions than the start position occur on the $\mathrm{x}$ vertices during the course of the game. In other applications one might need to initialize the game with other configurations than $\alpha^{-1}(0)$. For this, we define the initialization gadget more generally for every valid configuration $\mathfrak{q}$.

The initialization gadget INIT $^{\mathfrak{q}}$ is built out of two switches $M^{1}$ and $M^{2}$, vertices $z$ in Spoiler's graph and $z_{1}, z_{2}$ in Duplicator's graph. The three vertices $z, z_{1}, z_{2}$ share one unique vertex color. Additionally, there are output boundary vertices $y_{j}^{i}$ of the usual form. The vertices $z, z_{1}, z_{2}$ and the boundary vertices are connected to $M^{1}$ and $M^{2}$ as shown in Figure 6 for a specific valid configuration $\mathfrak{q}=(\mathfrak{a}, \mathfrak{b}, \emptyset)$. Lemma 11 (i)-(iii) provides the strategies on INIT $^{\mathfrak{q}}$. The main property is that Spoiler can reach the start position $\mathfrak{q}$ at the boundary (i) and Duplicator has a corresponding counter strategy (ii) in this situation. Furthermore, if an arbitrary position occurs at the boundary during the game, Duplicator has a strategy to survive (iii). 
Lemma 11. (A slightly more general version of Lemma 7) For every valid configuration $\mathfrak{q}=(\mathfrak{a}, \mathfrak{b}, \emptyset)$ the following holds in the existential $(\mathbf{k}+1)$-pebble game on INIT $^{q}$ :

(i) Spoiler can reach $\mathfrak{q}$ on the output.

(ii) There is a winning strategy $\mathcal{I}^{\text {init }}$ for Duplicator with boundary function $h_{\mathfrak{q}}^{y}$.

(iii) For every (valid or invalid) configuration $\mathfrak{q}^{\prime}$ there is a critical strategy $\mathcal{I}_{\mathfrak{q}^{\prime}}^{\text {init }}$ with boundary function $h_{\mathfrak{q}^{\prime}}^{y}$ and $\operatorname{crit}\left(\mathcal{I}_{\mathfrak{q}^{\prime}}^{\text {init }}\right) \subseteq \mathcal{I}^{\text {init }}$.

Spoiler's strategy is quite simple. First he pebbles $z$. Duplicator has to answer with either $z_{1}$ or $z_{2}$. Then Spoiler can reach $\left\{\left(x_{\mathfrak{a}(i)}^{i}, x_{\mathfrak{b}(i)}^{i}\right) \mid i \in[\mathrm{k}]\right\}$ by pebbling through either $M^{1}$ or $M^{2}$. To construct the strategies for Duplicator, we can combine the strategies of the switches $M^{1}$ and $M^{2}$ such that she plays an input strategy on one switch and a restart or output strategy on the other switch. Assume that Spoiler reaches a critical position on the switch where Duplicator plays the input strategy, say $M^{1}$. Duplicator can now flip the strategies such that she plays a restart or output strategy on $M^{1}$, depending on which kind of critical position Spoiler has reached, and an input strategy on $M^{2}$.

Proof (Proof of Lemma 11). We start with developing the strategy for Spoiler (i). First, Spoiler pebbles $z$. Duplicator has to response with either $z_{1}$ or $z_{2}$. Depending on Duplicator's choice, Spoiler can reach either $\left\{\left(a_{\mathfrak{a}(i)}^{i}, a_{\mathfrak{b}(i)}^{i}\right) \mid i \in[\mathbf{k}]\right\}$ or $\left\{\left(b_{\mathfrak{a}(i)}^{i}, b_{\mathfrak{b}(i)}^{i}\right) \mid i \in[\mathrm{k}]\right\}$. By Lemma 6 (i) Spoiler reaches $\left\{\left(c_{\mathfrak{a}(i)}^{i}, c_{\mathfrak{b}(i)}^{i}\right) \mid i \in[\mathrm{k}]\right\}$ $\left(\left\{\left(d_{\mathfrak{a}(i)}^{i}, d_{\mathfrak{b}(i)}^{i}\right) \mid i \in[\mathrm{k}]\right\}\right)$ and from there he can reach the position $\left\{\left(y_{\mathfrak{a}(i)}^{i}, y_{\mathfrak{b}(i)}^{i}\right) \mid\right.$ $i \in[\mathrm{k}]\}$. For Duplicator's strategies we start with a discussion of possible moves outside of the switches. At the top of the gadget Duplicator can map $z$ to $z_{1}$ and is then forced to answer with $h_{\mathfrak{q}}^{a}$ at the input of $M^{1}$ and for some $R \subseteq[\mathrm{k}]$ with $h_{(\mathfrak{a}, \mathfrak{b}, R)}^{b}$ at the input of $M^{2}$. On the other hand, Duplicator can map $z$ to $z_{2}$ and play according to $h_{(\mathfrak{a}, \mathfrak{b}, R)}^{a}$ and $h_{\mathfrak{q}}^{b}$. At the bottom of the switch the following three combinations define partial homomorphisms for all configurations $\mathfrak{q}^{\prime}$ :

$$
\begin{gathered}
h_{\mathbf{0}}^{c} \cup h_{\mathbf{0}}^{d} \cup h_{\mathfrak{q}^{\prime}}^{y} \\
h_{\mathfrak{q}}^{c} \cup h_{\mathbf{0}}^{d} \cup h_{\mathfrak{q}}^{y} \\
h_{\mathbf{0}}^{c} \cup h_{\mathfrak{q}}^{d} \cup h_{\mathfrak{q}}^{y}
\end{gathered}
$$

Now we can combine these partial strategies with the strategies on the switches described in Lemma 6. In strategy $\mathcal{I}_{t, \mathfrak{q}^{\prime}}^{\text {in } i}$ Duplicator plays an input strategy on switch $i$, a restart strategy on the other switch and according to an arbitrary configuration $\mathfrak{q}^{\prime}$ on the $y$-block. These strategies were combined to the critical strategy $\mathcal{I}_{\mathfrak{q}^{\prime}}^{\text {init }}$ described in (iii).

$$
\begin{aligned}
& \mathcal{I}_{t, \mathfrak{q}^{\prime}}^{\text {in-1 }}:=\wp\left(\left\{\left(z, z_{1}\right)\right\}\right) \uplus \mathcal{H}_{\mathfrak{q}}^{\text {in }}\left\langle M^{1}\right\rangle \uplus \mathcal{H}_{(\mathfrak{a}, \mathfrak{b},\{t\})}^{\text {restart }}\left\langle M^{2}\right\rangle \uplus \wp\left(h_{\mathfrak{q}^{\prime}}^{y}\right) \\
& \mathcal{I}_{t, \mathfrak{q}^{\prime}}^{\text {in-2 }}:=\wp\left(\left\{\left(z, z_{2}\right)\right\}\right) \uplus \mathcal{H}_{(\mathfrak{a}, \mathfrak{b},\{t\})}^{\text {restart }}\left\langle M^{1}\right\rangle \uplus \mathcal{H}_{\mathfrak{q}}^{\text {in }}\left\langle M^{2}\right\rangle \uplus \wp\left(h_{\mathfrak{q}^{\prime}}^{y}\right) \\
& \mathcal{I}_{\mathfrak{q}^{\prime}}^{\text {init }}:=\bigcup_{t \in[\mathbf{k}]}\left(\mathcal{I}_{t, \mathfrak{q}^{\prime}}^{\text {in-1 }} \cup \mathcal{I}_{t, \mathfrak{q}^{\prime}}^{\text {in-2 }}\right)
\end{aligned}
$$


All critical positions of $\mathcal{I}_{t, \mathfrak{q}^{\prime}}^{\mathrm{in} i}$ are restart or output critical positions on the switch $M^{i}$. By Lemma 6.(iv).(b) every restart critical position of $\mathcal{I}_{t, \mathfrak{q}^{\prime}}^{\text {in-1 }}$ is contained

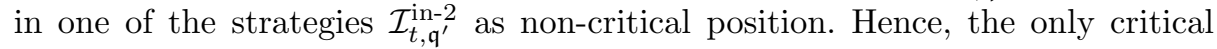
positions $\operatorname{crit}\left(\mathcal{I}_{\mathfrak{q}^{\prime}}^{\text {init }}\right)$ of the combined strategy are output critical positions on the switches. These output critical positions will be contained in the strategies $\mathcal{I}^{\text {init-i }}$ where Duplicator plays an output strategy on switch $i$. Together with $\mathcal{I}_{\mathfrak{q}}^{\text {init }}$ they form the winning strategy $\mathcal{I}^{\text {init }}$ from (ii).

$$
\begin{aligned}
\mathcal{I}^{\text {init-1 }} & :=\wp\left(\left\{\left(z, z_{2}\right)\right\}\right) \uplus \mathcal{H}_{\mathfrak{q}}^{\text {out }}\left\langle M^{1}\right\rangle \uplus \mathcal{H}_{\mathfrak{q}}^{\text {in }}\left\langle M^{2}\right\rangle \uplus \wp\left(h_{\mathfrak{q}}^{y}\right) \\
\mathcal{I}^{\text {init-2 }} & :=\wp\left(\left\{\left(z, z_{1}\right)\right\}\right) \uplus \mathcal{H}_{\mathfrak{q}}^{\text {in }}\left\langle M^{1}\right\rangle \uplus \mathcal{H}_{\mathfrak{q}}^{\text {out }}\left\langle M^{2}\right\rangle \uplus \wp\left(h_{\mathfrak{q}}^{y}\right) \\
\mathcal{I}^{\text {init }} & :=\mathcal{I}^{\text {init-1 }} \cup \mathcal{I}^{\text {init- }} \cup \mathcal{I}_{\mathfrak{q}}^{\text {init }}
\end{aligned}
$$

$\mathcal{I}^{\text {init }}$ is a union of critical strategies with boundary function $h_{\mathfrak{q}}^{y}$. To prove that $\mathcal{I}^{\text {init }}$ is indeed a winning strategy on the gadget, we show that every critical position of one strategy is contained as non-critical position in another strategy. Critical positions are inside the input strategy $\mathcal{H}_{\mathfrak{q}}^{\text {in }}$ on one of the switches. By Lemma 6.(iv) they are either contained in an output or restart strategy on the corresponding switch. Hence, all restart critical positions on $M^{1}$ and $M^{2}$ are contained in $\mathcal{I}_{\mathfrak{q}}^{\text {init }}$ and all output critical positions on $M^{1}\left(M^{2}\right)$ are contained in $\mathcal{I}^{\text {init-1 }}\left(\mathcal{I}^{\text {init-2}}\right)$. Recall the notation $\widehat{\mathcal{S}}:=\mathcal{S} \backslash \operatorname{crit}(\mathcal{S})$, by Lemma 6 (iv) we get:

$$
\begin{aligned}
\operatorname{crit}\left(\mathcal{I}_{R, \mathfrak{q}^{\prime}}^{\text {in-2 }}\right) & =\operatorname{crit}\left(\mathcal{I}^{\text {init-1 }}\right)=\operatorname{crit}\left(\mathcal{H}_{\mathfrak{q}}^{\text {in }}\left\langle M^{2}\right\rangle\right) \\
& \subseteq \mathcal{H}_{\mathfrak{q}}^{\text {out }}\left\langle M^{2}\right\rangle \cup \bigcup_{t \in[\mathrm{k}]} \mathcal{H}_{(\mathfrak{q},\{t\})}^{\text {restart }}\left\langle M^{2}\right\rangle \\
& \subseteq \widehat{\mathcal{I}}^{\text {init-2 }} \cup \bigcup_{t \in[\mathrm{k}]} \widehat{\mathcal{I}}_{\{t\}, \mathfrak{q}}^{\text {in-1 }}, \\
\operatorname{crit}\left(\mathcal{I}_{R, \mathfrak{q}^{\prime}}^{\text {in-1 }}\right) & =\operatorname{crit}\left(\mathcal{I}^{\text {init-2 }}\right)=\operatorname{crit}\left(\mathcal{H}_{\mathfrak{q}}^{\text {in }}\left\langle M^{1}\right\rangle\right) \\
& \subseteq \mathcal{H}_{\mathfrak{q}}^{\text {out }}\left\langle M^{1}\right\rangle \cup \bigcup_{t \in[k]} \mathcal{H}_{(\mathfrak{q},\{t\})}^{\text {restart }}\left\langle M^{1}\right\rangle \\
& \subseteq \widehat{\mathcal{I}}^{\text {init-1 }} \cup \bigcup_{t \in[k]} \widehat{\mathcal{I}}_{\{t\}, \mathfrak{q}}^{\text {in-2 }} .
\end{aligned}
$$

Hence, $\operatorname{crit}\left(\mathcal{I}_{\mathfrak{q}^{\prime}}^{\text {init }}\right) \subseteq \mathcal{I}^{\text {init }}$ and $\mathcal{I}^{\text {init }}$ is a winning strategy. 